\title{
Speciation in Multidimensional Evolutionary Space
}

András Vukics (vukics@optics.szfki.kfki.hu)

János Asbóth (asboth@optics.szfki.kfki.hu)

Géza Meszéna (geza@angel.elte.hu)

\section{Approved by}

Ulf Dieckmann (dieckmann@iiasa.ac.at)

Project Leader, Adaptive Dynamics Network

April 2004 


\section{IIASA STUdIES IN ADAPTIVE DYNAMICS No. 86}

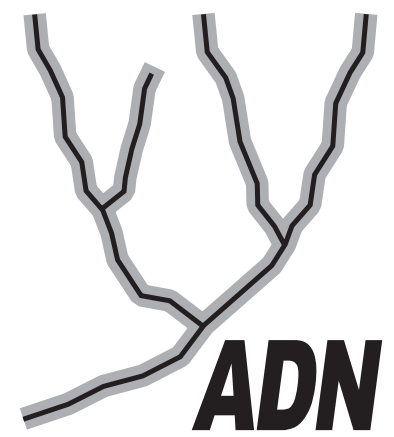

The Adaptive Dynamics Network at IIASA fosters the development of new mathematical and conceptual techniques for understanding the evolution of complex adaptive systems.

Focusing on these long-term implications of adaptive processes in systems of limited growth, the Adaptive Dynamics Network brings together scientists and institutions from around the world with IIASA acting as the central node.

Scientific progress within the network is collected in the IIASA Studies in Adaptive Dynamics series.

No. 1 Metz JAJ, Geritz SAH, Meszéna G, Jacobs FJA, van Heerwaarden JS: Adaptive Dynamics: A Geometrical Study of the Consequences of Nearly Faithful Reproduction. IIASA Working Paper WP-95-099 (1995). van Strien SJ, Verduyn Lunel SM (eds): Stochastic and Spatial Structures of Dynamical Systems, Proceedings of the Royal Dutch Academy of Science (KNAW Verhandelingen), North Holland, Amsterdam, pp. 183-231 (1996).

No. 2 Dieckmann U, Law R: The Dynamical Theory of Coevolution: A Derivation from Stochastic Ecological Processes. IIASA Working Paper WP-96-001 (1996). Journal of Mathematical Biology 34:579-612 (1996).

No. 3 Dieckmann U, Marrow P, Law R: Evolutionary Cycling of Predator-PreyInteractions: Population Dynamics and the Red Queen. IIASA Preprint (1995). Journal of Theoretical Biology 176:91-102 (1995).

No. 4 Marrow P, Dieckmann U, Law R: Evolutionary Dynamics of Predator-Prey Systems: An Ecological Perspective. IIASA Working Paper WP-96-002 (1996). Journal of Mathematical Biology 34:556-578 (1996).

No. 5 Law R, Marrow P, Dieckmann U: On Evolution under Asymmetric Competition. IIASA Working Paper WP-96-003 (1996). Evolutionary Ecology 11:485-501 (1997).

No. 6 Metz JAJ, Mylius SD, Diekmann O: When Does Evolution Optimize? On the Relation Between Types of Density Dependence and Evolutionarily Stable Life History Parameters. IIASA Working Paper WP-96-004 (1996).

No. 7 Ferrière R, Gatto M: Lyapunov Exponents and the Mathematics of Invasion in Oscillatory or Chaotic Populations. Theoretical Population Biology 48:126-171 (1995).

No. 8 Ferrière R, Fox GA: Chaos and Evolution. IIASA Preprint (1996). Trends in Ecology and Evolution 10:480485 (1995).

No. 9 Ferrière R, Michod RE: The Evolution of Cooperation in Spatially Heterogeneous Populations. IIASA Working Paper WP-96-029 (1996). The American Naturalist 147:692717 (1996).

No. 10 van Dooren TJM, Metz JAJ: Delayed Maturation in Temporally Structured Populations with Non-Equilibrium Dynamics. IIASA Working Paper WP-96-070 (1996). Journal of Evolutionary Biology 11:41-62 (1998).
No. 11 Geritz SAH, Metz JAJ, Kisdi É, Meszéna G: The Dynamics of Adaptation and Evolutionary Branching. IIASA Working Paper WP-96-077 (1996). Physical Review Letters 78:2024-2027 (1997).

No. 12 Geritz SAH, Kisdi É, Meszéna G, Metz JAJ: Evolutionary Singular Strategies and the Adaptive Growth and Branching of the Evolutionary Tree. IIASA Working Paper WP-96-114 (1996). Evolutionary Ecology 12:35-57 (1998).

No. 13 Heino M, Metz JAJ, Kaitala V: Evolution of Mixed Maturation Strategies in Semelparous Life-Histories: The Crucial Role of Dimensionality of Feedback Environment. IIASA Working Paper WP-96-126 (1996). Philosophical Transactions of the Royal Society of London Series B 352:1647-1655 (1997).

No. 14 Dieckmann U: Can Adaptive Dynamics Invade? IIASA Working Paper WP-96-152 (1996). Trends in Ecology and Evolution 12:128-131 (1997).

No. 15 Meszéna G, Czibula I, Geritz SAH: Adaptive Dynamics in a 2-Patch Environment: A Simple Model for Allopatric and Parapatric Speciation. IIASA Interim Report IR-97-001 (1997). Journal of Biological Systems 5:265-284 (1997).

No. 16 Heino M, Metz JAJ, Kaitala V: The Enigma of Frequency-Dependent Selection. IIASA Interim Report IR97-061 (1997). Trends in Ecology and Evolution 13:367-370 (1998).

No. 17 Heino M: Management of Evolving Fish Stocks. IIASA Interim Report IR-97-062 (1997). Canadian Journal of Fisheries and Aquatic Sciences 55:1971-1982 (1998).

No. 18 Heino M: Evolution of Mixed Reproductive Strategies in Simple Life-History Models. IIASA Interim Report IR-97063 (1997).

No. 19 Geritz SAH, van der Meijden E, Metz JAJ: Evolutionary Dynamics of Seed Size and Seedling Competitive Ability. IIASA Interim Report IR-97-071 (1997). Theoretical Population Biology 55:324-343 (1999).

No. 20 Galis F, Metz JAJ: Why Are There So Many Cichlid Species? On the Interplay of Speciation and Adaptive Radiation. IIASA Interim Report IR-97-072 (1997). Trends in Ecology and Evolution 13:1-2 (1998). 
No. 21 Boerlijst MC, Nowak MA, Sigmund K: Equal Pay for all Prisoners/ The Logic of Contrition. IIASA Interim Report IR-97-073 (1997). American Mathematical Society Monthly 104:303-307 (1997). Journal of Theoretical Biology 185:281-293 (1997).

No. 22 Law R, Dieckmann U: Symbiosis Without Mutualism and the Merger of Lineages in Evolution. IIASA Interim Report IR-97-074 (1997). Proceedings of the Royal Society of London Series B 265:1245-1253 (1998).

No. 23 Klinkhamer PGL, de Jong TJ, Metz JAJ: Sex and Size in Cosexual Plants. IIASA Interim Report IR-97-078 (1997). Trends in Ecology and Evolution 12:260-265 (1997).

No. 24 Fontana W, Schuster P: Shaping Space: The Possible and the Attainable in RNA Genotype-Phenotype Mapping. IIASA Interim Report IR-98-004 (1998). Journal of Theoretical Biology 194:491-515 (1998).

No. 25 Kisdi É, Geritz SAH: Adaptive Dynamics in Allele Space: Evolution of Genetic Polymorphism by Small Mutations in a Heterogeneous Environment. IIASA Interim Report IR-98-038 (1998). Evolution 53:993-1008 (1999).

No. 26 Fontana W, Schuster P: Continuity in Evolution: On the Nature of Transitions. IIASA Interim Report IR-98-039 (1998). Science 280:1451-1455 (1998).

No. 27 Nowak MA, Sigmund K: Evolution of Indirect Reciprocity by Image Scoring/ The Dynamics of Indirect Reciprocity. IIASA Interim Report IR-98-040 (1998). Nature 393:573-577 (1998). Journal of Theoretical Biology 194:561574 (1998).

No. 28 Kisdi É: Evolutionary Branching Under Asymmetric Competition. IIASA Interim Report IR-98-045 (1998). Journal of Theoretical Biology 197:149-162 (1999).

No. 29 Berger U: Best Response Adaptation for Role Games. IIASA Interim Report IR-98-086 (1998).

No. 30 van Dooren TJM: The Evolutionary Ecology of Dominance-Recessivity. IIASA Interim Report IR-98-096 (1998). Journal of Theoretical Biology 198:519-532 (1999).

No. 31 Dieckmann U, O'Hara B, Weisser W: The Evolutionary Ecology of Dispersal. IIASA Interim Report IR-98-108 (1998). Trends in Ecology and Evolution 14:88-90 (1999).

No. 32 Sigmund K: Complex Adaptive Systems and the Evolution of Reciprocation. IIASA Interim Report IR-98-100 (1998). Ecosystems 1:444-448 (1998).

No. 33 Posch M, Pichler A, Sigmund K: The Efficiency of Adapting Aspiration Levels. IIASA Interim Report IR-98103 (1998). Proceedings of the Royal Society London Series B 266:1427-1435 (1999).

No. 34 Mathias A, Kisdi É: Evolutionary Branching and Coexistence of Germination Strategies. IIASA Interim Report IR-99-014 (1999).

No. 35 Dieckmann U, Doebeli M: On the Origin of Species by Sympatric Speciation. IIASA Interim Report IR-99-013 (1999). Nature 400:354-357 (1999).

No. 36 Metz JAJ, Gyllenberg M: How Should We Define Fitness in Structured Metapopulation Models? Including an Application to the Calculation of Evolutionarily Stable Dispersal Strategies. IIASA Interim Report IR-99-019 (1999). Proceedings of the Royal Society of London Series B 268:499508 (2001)
No. 37 Gyllenberg M, Metz JAJ: On Fitness in Structured Metapopulations. IIASA Interim Report IR-99-037 (1999). Journal of Mathematical Biology 43:545-560 (2001).

No. 38 Meszéna G, Metz JAJ: Species Diversity and Population Regulation: The Importance of Environmental Feedback Dimensionality. IIASA Interim Report IR-99-045 (1999).

No. 39 Kisdi É, Geritz SAH: Evolutionary Branching and Sympatric Speciation in Diploid Populations. IIASA Interim Report IR-99-048 (1999).

No. 40 Ylikarjula J, Heino M, Dieckmann U: Ecology and Adaptation of Stunted Growth in Fish. IIASA Interim Report IR-99-050 (1999). Evolutionary Ecology 13:433-453 (1999).

No. 41 Nowak MA, Sigmund K: Games on Grids. IIASA Interim Report IR-99-038 (1999). Dieckmann U, Law R, Metz JAJ (eds): The Geometry of Ecological Interactions: Simplifying Spatial Complexity, Cambridge University Press, Cambridge, UK, pp. 135-150 (2000).

No. 42 Ferrière R, Michod RE: Wave Patterns in Spatial Games and the Evolution of Cooperation. IIASA Interim Report IR-99-041 (1999). Dieckmann U, Law R, Metz JAJ (eds): The Geometry of Ecological Interactions: Simplifying Spatial Complexity, Cambridge University Press, Cambridge, UK, pp. 318-332 (2000).

No. 43 Kisdi É, Jacobs FJA, Geritz SAH: Red Queen Evolution by Cycles of Evolutionary Branching and Extinction. IIASA Interim Report IR-00-030 (2000). Selection 2:161176 (2001).

No. 44 Meszéna G, Kisdi É, Dieckmann U, Geritz SAH, Metz JAJ: Evolutionary Optimisation Models and Matrix Games in the Unified Perspective of Adaptive Dynamics. IIASA Interim Report IR-00-039 (2000). Selection 2:193-210 (2001).

No. 45 Parvinen K, Dieckmann U, Gyllenberg M, Metz JAJ: Evolution of Dispersal in Metapopulations with Local Density Dependence and Demographic Stochasticity. IIASA Interim Report IR-00-035 (2000). Journal of Evolutionary Biology $16: 143-153$ (2003).

No. 46 Doebeli M, Dieckmann U: Evolutionary Branching and Sympatric Speciation Caused by Different Types of Ecological Interactions. IIASA Interim Report IR-00-040 (2000). The American Naturalist 156:S77-S101 (2000).

No. 47 Heino M, Hanski I: Evolution of Migration Rate in a Spatially Realistic Metapopulation Model. IIASA Interim Report IR-00-044 (2000). The American Naturalist 157:495511 (2001).

No. 48 Gyllenberg M, Parvinen K, Dieckmann U: Evolutionary Suicide and Evolution of Dispersal in Structured Metapopulations. IIASA Interim Report IR-00-056 (2000). Journal of Mathematical Biology 45:79-105 (2002).

No. 49 van Dooren TJM: The Evolutionary Dynamics of Direct Phenotypic Overdominance: Emergence Possible, Loss Probable. IIASA Interim Report IR-00-048 (2000). Evolution 54: 1899-1914 (2000).

No. 50 Nowak MA, Page KM, Sigmund K: Fairness Versus Reason in the Ultimatum Game. IIASA Interim Report IR00-57 (2000). Science 289:1773-1775 (2000).

No. 51 de Feo O, Ferrière R: Bifurcation Analysis of Population Invasion: On-Off Intermittency and Basin Riddling. IIASA Interim Report IR-00-074 (2000). International Journal of Bifurcation and Chaos 10:443-452 (2000). 
No. 52 Heino M, Laaka-Lindberg S: Clonal Dynamics and Evolution of Dormancy in the Leafy Hepatic Lophozia Silvicola. IIASA Interim Report IR-01-018 (2001). Oikos 94:525-532 (2001).

No. 53 Sigmund K, Hauert C, Nowak MA: Reward and Punishment in Minigames. IIASA Interim Report IR-01-031 (2001). Proceedings of the National Academy of Sciences of the USA 98:10757-10762(2001).

No. 54 Hauert C, De Monte S, Sigmund K, Hofbauer J: Oscillations in Optional Public Good Games. IIASA Interim Report IR-01-036 (2001).

No. 55 Ferrière R, Le Galliard J: Invasion Fitness and Adaptive Dynamics in Spatial Population Models. IIASA Interim Report IR-01-043 (2001). Clobert J, Dhondt A, Danchin E, Nichols J (eds): Dispersal, Oxford University Press, pp. 57-79 (2001).

No. 56 de Mazancourt C, Loreau M, Dieckmann U: Can the Evolution of Plant Defense Lead to Plant-Herbivore Mutualism. IIASA Interim Report IR-01-053 (2001). The American Naturalist 158: 109-123 (2001).

No. 57 Claessen D, Dieckmann U: Ontogenetic Niche Shifts and Evolutionary Branching in Size-Structured Populations. IIASA Interim Report IR-01-056 (2001). Evolutionary Ecology Research 4:189-217 (2002).

No. 58 Brandt H: Correlation Analysis of Fitness Landscapes. IIASA Interim Report IR-01-058 (2001).

No. 59 Dieckmann U: Adaptive Dynamics of Pathogen-Host Interacations. IIASA Interim Report IR-02-007 (2002). Dieckmann U, Metz JAJ, Sabelis MW, Sigmund K (eds): Adaptive Dynamics of Infectious Diseases: In Pursuit of Virulence Management, Cambridge University Press, Cambridge, UK, pp. 39-59 (2002).

No. 60 Nowak MA, Sigmund K: Super- and Coinfection: The Two Extremes. IIASA Interim Report IR-02-008 (2002). Dieckmann U, Metz JAJ, Sabelis MW, Sigmund K (eds): Adaptive Dynamics of Infectious Diseases: In Pursuit of Virulence Management, Cambridge University Press, Cambridge, UK, pp. 124-137 (2002).

No. 61 Sabelis MW, Metz JAJ: Perspectives for Virulence Management: Relating Theory to Experiment. IIASA Interim Report IR-02-009 (2002). Dieckmann U, Metz JAJ, Sabelis MW, Sigmund K (eds): Adaptive Dynamics of Infectious Diseases: In Pursuit of Virulence Management, Cambridge University Press, Cambridge, UK, pp. 379-398 (2002).

No. 62 Cheptou P, Dieckmann U: The Evolution of SelfFertilization in Density-Regulated Populations . IIASA Interim Report IR-02-024 (2002). Proceedings of the Royal Society of London Series B 269:1177-1186 (2002).

No. 63 Bürger R: Additive Genetic Variation Under Intraspecific Competition and Stabilizing Selection: A Two-Locus Study. IIASA Interim Report IR-02-013 (2002). Theoretical Population Biology 61:197-213 (2002).

No. 64 Hauert C, De Monte S, Hofbauer J, Sigmund K: Volunteering as Red Queen Mechanism for Co-operation in Public Goods Games. IIASA Interim Report IR-02-041 (2002). Science 296:1129-1132(2002).

No. 65 Dercole F, Ferrière R, Rinaldi S: Ecological Bistability and Evolutionary Reversals under Asymmetrical Competition. IIASA Interim Report IR-02-053 (2002). Evolution 56:1081-1090 (2002).
No. 66 Dercole F, Rinaldi S: Evolution of Cannibalistic Traits: Scenarios Derived from Adaptive Dynamics. IIASA Interim Report IR-02-054 (2002). Theoretical Population Biology 62:365-374 (2002).

No. 67 Bürger R, Gimelfarb A: Fluctuating Environments and the Role of Mutation in Maintaining Quantitative Genetic Variation. IIASA Interim Report IR-02-058 (2002). Genetical Research 80:31-46 (2002).

No. 68 Bürger R: On a Genetic Model of Intraspecific Competition and Stabilizing Selection. IIASA Interim Report IR02-062 (2002). Amer. Natur. 160:661-682 (2002).

No. 69 Doebeli M, Dieckmann U: Speciation Along Environmental Gradients. IIASA Interim Report IR-02-079 (2002). Nature 421:259-264 (2003).

No. 70 Dercole F, Irisson J, Rinaldi S: Bifurcation Analysis of a Prey-Predator Coevolution Model. IIASA Interim Report IR-02-078 (2002). SIAM Journal on Applied Mathematics 63:1378-1391 (2003).

No. 71 Le Galliard J, Ferrière R, Dieckmann U: The Adaptive Dynamics of Altruism in Spatially Heterogeneous Populations. IIASA Interim Report IR-03-006 (2003). Evolution 57:1-17 (2003).

No. 72 Taborsky B, Dieckmann U, Heino M: Unexpected Discontinuities in Life-History Evolution under SizeDependent Mortality. IIASA Interim Report IR-03-004 (2003). Proceedings of the Royal Society of London Series B 270:713-721 (2003).

No. 73 Gardmark A, Dieckmann U, Lundberg P: LifeHistory Evolution in Harvested Populations: The Role of Natural Predation. IIASA Interim Report IR-03-008 (2003). Evolutionary Ecology Research 5:239-257 (2003).

No. 74 Mizera F, Meszéna G: Spatial Niche Packing, Character Displacement and Adaptive Speciation Along an Environmental Gradient. IIASA Interim Report IR-03-062 (2003). Evolutionary Ecology Research 5: 363-382 (2003).

No. 75 Dercole F: Remarks on Branching-Extinction Evolutionary Cycles. IIASA Interim Report IR-03-075 (2003). Journal of Mathematical Biology 47: 569-580 (2003).

No. 76 Hofbauer J, Sigmund K: Evolutionary Game Dynamics. IIASA Interim Report IR-03-078 (2003). Bulletin of the American Mathematical Society 40: 479-519 (2003).

No. 77 Ernande B, Dieckmann U, Heino M: Adaptive Changes in Harvested Populations: Plasticity and Evolution of Age and Size at Maturation. IIASA Interim Report IR-03058 (2003).

No. 78 Hanski I, Heino M: Metapopulation-Level Adaptation of Insect Host Plant Preference and Extinction-Colonization Dynamics in Heterogeneous Landscapes. IIASA Interim Report IR-03-028 (2003). Theoretical Population Biology 63:309-338 (2003).

No. 79 van Doorn G, Dieckmann U, Weissing FJ: Sympatric Speciation by Sexual Selection: A Critical Re-Evaluation. IIASA Interim Report IR-04-003 (2004).

No. 80 Egas M, Dieckmann U, Sabelis MW: Evolution Restricts the Coexistence of Specialists and Generalists - the Role of Trade-off Structure. IIASA Interim Report IR-04-004 (2004). 
No. 81 Ernande B, Dieckmann U: The Evolution of Phenotypic Plasticity in Spatially Structured Environments: Implications of Intraspecific Competition, Plasticity Costs, and Environmental Characteristics. IIASA Interim Report IR-04-006 (2004). Journal of Evolutionary Biology (2004).

No. 82 Cressman R, Hofbauer J: Measure Dynamics on a One-Dimensional Continuous Trait Space: Theoretical Foundations for Adaptive Dynamics. IIASA Interim Report IR04-016 (2004).

No. 83 Cressman R: Dynamic Stability of the Replicator Equation with Continuous Strategy Space. IIASA Interim Report IR-04-017 (2004).
No. 84 Ravigné V, Olivieri I, Dieckmann U: Implications of Habitat Choice for Protected Polymorphisms. IIASA Interim Report IR-04-005 (2004). Evolutionary Ecology Research 6: 125-145 (2004).

No. 85 Nowak MA, Sigmund K: Evolutionary Dynamics of Biological Games. IIASA Interim Report IR-04-013 (2004). Science 303: 793-799 (2004).

No. 86 Vukics A, Asbóth J, Meszéna G: Speciation in Multidimensional Evolutionary Space. IIASA Interim Report IR-04-028 (2004).

Issues of the IIASA Studies in Adaptive Dynamics series can be obtained at www.iiasa.ac.at/Research/ADN/Series.html or by writing to adn@iiasa.ac.at. 


\section{Contents}

1 Introduction: Environmental feedback and adaptive speciation $\quad 1$

2 Theoretical background 2

2.1 Environmental feedback . . . . . . . . . . . . . . . . . . 2

2.2 Assumptions about modelling evolution . . . . . . . . . . . . 2

2.3 Directional evolution . . . . . . . . . . . . . . . . . . . 3

2.4 Singular strategies . . . . . . . . . . . . . . . . . . 4

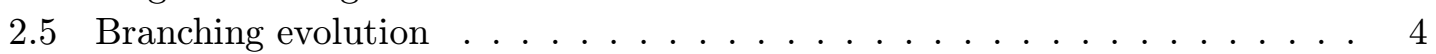

2.6 Local coexistence . . . . . . . . . . . . . . . . . . . . . . 4

3 Model 5

4 Results $\quad 7$

4.1 The pattern of phase transitions to evolutionary branching $\ldots \ldots \ldots$. . 7

4.2 The branching pattern . . . . . . . . . . . . . . 7

4.3 Statistics of the branching types . . . . . . . . . . . . 12

5 Conclusions $\quad 21$ 


\begin{abstract}
Adaptive dynamics in two dimensional phenotype space is investigated by computer simulation. The model assumes Lotka-Voltera type competition and a stochastic mutation process. The carrying capacity has a single maximum in the origin of the strategy space and the competition coefficient decreases with strategy difference. Evolutionary branching, an asexual analogue of adaptive speciation, is observed with suitable parameters. The branching at the singular point, which is a fixed point of the directional evolution, may occur into two or three, but no more directions. Further branchings may occur after the initial separation. The probability of three-branching is studied as a function of several parameters. We conclude that the two-way branching is the predominant mode of adaptive speciation.
\end{abstract}




\title{
About the Authors
}

\author{
András Vukics \\ Department of Nonlinear and Quantum Optics, \\ Institute for Solid State Physics and Optics, \\ Hungarian Academy of Sciences, \\ P.O. Box 49 H-1525 Budapest, Hungary \\ and \\ Department of Biological Physics, \\ Eötvös University, Pázmány Péter sétány 1A, \\ H-1117 Budapest, Hungary \\ János Asbóth \\ Department of Nonlinear and Quantum Optics, \\ Institute for Solid State Physics and Optics, \\ Hungarian Academy of Sciences, \\ P.O. Box 49 H-1525 Budapest, Hungary \\ and \\ Department of Biological Physics, \\ Eötvös University, Pázmány Péter sétány 1A, \\ H-1117 Budapest, Hungary \\ Géza Meszéna \\ Department of Biological Physics, \\ Eötvös University, Pázmány Péter sétány 1A, \\ H-1117 Budapest, Hungary \\ and \\ Adaptive Dynamics Network, \\ International Institute for Applied \\ Systems Analysis, \\ A-2361 Laxenburg, Austria
}

\section{Acknowledgement}

We thank Ulf Dieckmann, Éva Kisdi, Stefan Geritz, Hans Metz and Peter Jagers for discussions. This work was financed from the OTKA grant T033097 and from the NWOOTKA grant 048.011.039. 


\title{
Speciation in Multidimensional Evolutionary Space
}

\author{
András Vukics \\ János Asbóth \\ Géza Meszéna
}

\section{Introduction: Environmental feedback and adaptive spe- ciation}

Adaptive speciation $[1,2,3,4,5,6,7,8,9,10,11,12,13]$ is the most parsimonious concept on the origin of a new species. It is a literal implementation of Darwin's idea of descent via a series of small adaptive modifications [14]. The tricky thing to understand is how evolution uphill on the adaptive landscape can lead to a diversity of species, as opposed to being stopped at a (local) maximum of the fitness.

An inherent feature of the Darwinian process provides the answer. Evolution modifies the environment and, in turn, the fitness function. Existence of this feedback is not an extra assumption to introduce for explaining the process of speciation. Instead, it is a mathematical precondition of the very existence of more than a single species. Without the feedback, parameter fine-tuning would be necessary to avoid the best species to outcompete the other ones. The general theory of the combined dynamics of the evolving population and of the changing environment was presented earlier for one dimensional (1D) evolutionary state space $[15,16,17]$, see also $[18,19,20,21]$. It was shown that evolution toward increasing fitness can converge easily into a minimum, instead of a maximum, of the fitness function $[22,23,24,25]$. Then, the emerging disruptive selection splits the population into two subpopulations and drives them to evolve away from each other $[16,17]$. The theory of "adaptive speciation" hypothesises that the very same disruptive selection results in the adaptive emergence of sexual isolation between the two types.

In some biological situations, emergence of new species clusters into a brief period and form a so called "adaptive radiation" [26, 27]. This phenomenon is very characteristic and in the middle of interest of speciation studies. Like speciation in general, adaptive radiation is also a matter of intense debates. In the context of adaptive speciation, the following question arises: Is it possible that a single event of evolutionary branching leads to more than two species?

The answer is a definite no for $1 \mathrm{D}$ evolutionary state space. Only two branches can appear in a single branching event in this case. However, this space is multidimensional in any real case. So, we have to ask: Does the multidimensionality affects the phenomenon of evolutionary branching in an essential way?

As we will see, a meaningful deterministic approximation is valid in a proper limit away from some fixed points, referred to as "singular": Mutations should be small and rare for this limit. Around the singular point, however, the evolutionary process remains inherently stochastic. Since the interfacing between the stochastic and deterministic regimes is difficult to handle analytically, the branching process should be studied numerically. An 
analytic argument says that, at most, $K+1$ branches can appear in a single branching event at a singular point, where $K$ is the number of dimensions of the evolutionary state space. This would allow high number of species to appear in a single event of speciation when the state-space dimensionality is high. However, the analytic argument does not ensure the existence of more-than-two-way branchings, and tells nothing about the relative rates of different types of branchings. These issues has remained to be checked by numerical experimentation, which is the main goal of the present paper. We are especially interested in the behaviour in the limit when the deterministic approximation is valid.

After summarising the available analytical insights in Sec. 2, we introduce a specific model in Sec. 3, and present results of numerical simulations for 2D state space in Sec. 4. We argue for the model-independent validity of our findings and discuss their biological consequences in Sec. 5 .

\section{Theoretical background}

\subsection{Environmental feedback}

To describe the evolution-environment feedback loop properly, one should derive the fitness function and the dynamics of evolution from the underlying population dynamics. This section summarises some of the theoretical results of $[15,16,17,28,29]$ about this connection.

We collect all the environmental variables involved in the feedback loop into the environmental interaction variable $\boldsymbol{I}[30,31]$. (For instance, concentrations of different resources are possible elements of $\boldsymbol{I}$.) The population dynamics of a specific species with size $n(t)$ at time $t$ can be written into the form

$$
\frac{d n}{d t}=r(\boldsymbol{x}, \boldsymbol{I}) \cdot n
$$

where the growth rate, or fitness $r$ is the difference between the rate of giving birth and the rate of death of an individual. The variable $\boldsymbol{x}$, which will be referred to as "strategy", represents the heritable properties of the species. At a fixed value of the $\boldsymbol{I}$, the function $\boldsymbol{x} \mapsto r(\boldsymbol{x}, \boldsymbol{I})$ represents the fitness landscape.

However, the assumption of constant $\boldsymbol{I}$ would lead to the absurd consequence of unlimited exponential growth. In the real world, growing population deteriorates the environment until the equilibrium environment $\boldsymbol{I}_{\boldsymbol{x}}$, characterised by $r\left(\boldsymbol{x}, \boldsymbol{I}_{\boldsymbol{x}}\right)=0$ is reached. (Only fixed-point attractors of the population dynamics are considered in this paper.) For the $L$ number of coexisting strategies $\boldsymbol{x}^{(1)}, \boldsymbol{x}^{(2)}, \ldots, \boldsymbol{x}^{(L)}$, the $L$ number of equilibrium conditions $r\left(\boldsymbol{x}^{(i)}, \boldsymbol{I}\right)=0$ should be satisfied. Generally, solvability of this set of equations implies the inequality $L \leq \operatorname{dim} \boldsymbol{I}$. This bound is referred to as the "principle of competitive exclusion" in ecology $[32,33,34,35,36]$. Note, however, that $\operatorname{dim} \boldsymbol{I}$ is often infinite.

\subsection{Assumptions about modelling evolution}

To study evolution via small steps, one should specify the set of possible species, the strategy space, as a continuum. Accordingly, we suppose that the strategy $\boldsymbol{x}$ is an $K$ dimensional continuous variable. Value of $\boldsymbol{x}$ is kept fixed during the life-time of an individual and inherited faithfully, except when mutation occurs. Only a finite number of different strategies are present at any given time. The list of the strategies changes with mutations and extinctions. 
We assume time-scale separation between population dynamics and evolution. Accordingly, whenever possible, we suppose that a new mutant arrives in the equilibrium environment set by the strategies that are already present. As the mutant population is initially small, its appearance does not change the environment $\boldsymbol{I}$ immediately. Consequently, the initial growth rate of the mutant of strategy $\boldsymbol{y}$ is $r_{\text {mut }}=r(\boldsymbol{y}, \boldsymbol{I})$, where $\boldsymbol{I}$ is the equilibrium environment set by the resident. That is, there is no environmental feedback operating on the mutant population, so its initial growth would be exponential in the deterministic approximation. However, because of the smallness of the emerging population, stochastic aspects are non-negligible.

Stochastic replication of independent individuals is analogous to the multiplication of neutrons in a nuclear chain reaction and many other types of self-replication. Such processes are described by the branching-process theory [37]. (Note that these "branching processes" have nothing to do with phenomenon of "evolutionary branching", which is a main issue of the present paper. The first one is concerned with the branching of lines of individual descent while the second one is concerned with the branching of evolutionary lines.) According to this theory, the process is sub-critical for $r_{\text {mut }}<0$, when the births cannot compensate for the deaths. The clone of mutants dies out with probability 1 in this case. In the supercritical situation, when births prevail over deaths $\left(r_{\text {mut }}>0\right)$, there is a positive chance for the long-term survival of the clone. (This probability is proportional to $r_{\text {mut }}$ for small $r_{\text {mut }}$ by linearisation.)

We suppose that the mutant clone has already achieved deterministic growth when it starts to modify the environment $\boldsymbol{I}$. In other words, individuals remain independent and the branching-process theory remains applicable during the stochastic phase. Moreover, we suppose that each evolutionary step is small, so the mutant strategy is almost identical to the strategy of its ancestor. This assumption leads to the picture of continuous and deterministic dynamics of the evolutionary process.

\subsection{Directional evolution}

The mutant strategy, which does not die out, invades the resident populations. Mutant invasion may result in ousting of its ancestor. This is especially the case when the mutational step-size is small and the "selection gradient", or "local fitness gradient"

$$
\boldsymbol{D}(\boldsymbol{x})=\left.\frac{\partial r(\boldsymbol{y}, \boldsymbol{I})}{\partial \boldsymbol{y}}\right|_{\boldsymbol{y}=\boldsymbol{x}}
$$

is different form zero [29]. Consecutive steps of such evolutionary replacements constitute a continuous evolution of the strategy.

The random process of substitutions can be approximated by a deterministic dynamics of evolution, provided that the mutations are rare and mutation steps are small. Dieckmann \& Law [28] established that the evolutionary dynamics of a single strategy $\boldsymbol{x}$ is

$$
\frac{d \boldsymbol{x}}{d t}=\frac{1}{2} \mu n \cdot \boldsymbol{C D}(\boldsymbol{x})
$$

The matrix $\boldsymbol{C}$ is the variance-covariance matrix of the difference vector between the mutant's and its ancestor's strategy. If the mutant strategy is distributed uniformly in the $\varepsilon$ neighbourhood of strategy $\boldsymbol{x}$, the covariance matrix becomes $\boldsymbol{C}=\frac{1}{2} \varepsilon^{2} \mathbf{1}$. (1 is the unit matrix.) This leads to the simplification

$$
\frac{d \boldsymbol{x}}{d t}=\gamma n \boldsymbol{D}(x)
$$

where $\gamma=\mu \varepsilon^{2} / 4$ contains the constant factors. 


\subsection{Singular strategies}

A strategy $\hat{\boldsymbol{x}}$ is referred to as a singular one, if $\boldsymbol{D}(\hat{\boldsymbol{x}})=0$, that is, if directional evolution ceases at $\hat{\boldsymbol{x}}$. This fixed point is stable under the dynamics (4) if and only if the Jacobian matrix

$$
\boldsymbol{J}=\left.\frac{\partial \boldsymbol{D}(\boldsymbol{x})}{\partial \boldsymbol{x}}\right|_{\boldsymbol{x}=\hat{\boldsymbol{x}}}=\left.\frac{\partial^{2} r\left(\boldsymbol{y}, \boldsymbol{I}_{\hat{\boldsymbol{x}}}\right)}{\partial \boldsymbol{y}^{2}}\right|_{\boldsymbol{y}=\hat{\boldsymbol{x}}}+\left.\frac{\partial r\left(\hat{\boldsymbol{x}}, \boldsymbol{I}_{\boldsymbol{x}}\right)}{\partial \boldsymbol{I}_{\boldsymbol{x}}} \cdot \frac{\partial \boldsymbol{I}_{\boldsymbol{x}}}{\partial \boldsymbol{x}}\right|_{\boldsymbol{x}=\hat{\boldsymbol{x}}}
$$

is negative definite $[28,38]$. A singular strategy with this kind of stability is referred to as a convergence stable one.

Note that a convergent stable singular strategy is not necessarily a local maximum of the fitness function. Negative definitiveness of the first term of Eq. (5) would correspond to a fitness maximum. The second term represents the change of the fitness gradient via the evolution-induced environmental change. It may result in a negative definite Jacobian even if the first term is not negative definite; that is, it is possible that the directional evolution converges to a singular strategy, which is not a local maximum of the fitness function $[22,23,24,25]$. Local maxima of the fitness are referred to as (locally) Evolutionary Stable Strategy, or ESS [39], because a mutant strategy that is similar to the resident, is unable to invade such a resident. (See also [40] for the intricacies of the ESS concept in relation to evolutionary game theory.)

\subsection{Branching evolution}

What happens if the evolutionary process converges to a singular point, which is convergent stable but not evolutionary stable? (In other words, what if evolution converges to a minimum of the fitness function?) It is not protected against mutations but cannot evolve away either. For one dimensional trait space it was shown that "evolutionary branching" is a necessary outcome of this situation $[16,17]$. Coexistence of two strategies, located on the opposite sides of the singular point, is always possible in the vicinity of this type of singularity. As soon as the coexistence is established, the selection forces acting on the two strategies on the opposite sides of the fitness minimum push them away from each other. Apart from the singular point the canonical equation (3) governs the evolution of each branch again. (The interaction between the branches have to be taken into account via the feedback variable $\boldsymbol{I}$.) New singular points may be reached and further branchings may occur [17].

No complete analytic theory of evolutionary branching is known for more than one dimensions. The main purpose of the paper is to investigate this situation.

\subsection{Local coexistence}

There is a bound on the branches appearing in a single branching event [15]. At most $K+1$ number of strategies can coexist in a $K$ dimensional strategy space in the vicinity of the singular strategy $\hat{\boldsymbol{x}}$.

To see this, we expand the fitness function into Taylor series around $\hat{\boldsymbol{x}}$. As the slope of the fitness is zero at $\hat{\boldsymbol{x}}$, one should consider the expansion up to second order to see the non-vanishing terms:

$$
r(\boldsymbol{x}, \boldsymbol{I})=\alpha(\boldsymbol{I})+\sum_{k=1}^{K} \beta_{k}(\boldsymbol{I})\left(x_{k}-\hat{x}_{k}\right)+\sum_{k, l=1}^{K} \gamma_{k l}(\boldsymbol{I})\left(x_{k}-\hat{x}_{k}\right)\left(x_{l}-\hat{x}_{l}\right)+\text { h.o.t. }
$$


The first two terms of (6) are zero for $\boldsymbol{I}=\boldsymbol{I}_{\hat{\boldsymbol{x}}}$. For the coexistence of strategies $\boldsymbol{x}^{(1)}, \boldsymbol{x}^{(2)}, \ldots, \boldsymbol{x}^{(L)}$, all near to $\hat{\boldsymbol{x}}$, the equilibrium conditions

$$
\begin{aligned}
r\left(\boldsymbol{x}^{(i)}, \boldsymbol{I}\right)=\alpha(\boldsymbol{I})+\sum_{k=1}^{K} \beta_{k}(\boldsymbol{I})( & \left.x_{k}^{(i)}-\hat{x}_{k}\right)+ \\
& +\sum_{k, l=1}^{K} \gamma_{k l}\left(\boldsymbol{I}_{\hat{\boldsymbol{x}}}\right)\left(x_{k}^{(i)}-\hat{x}_{k}\right)\left(x_{l}^{(i)}-\hat{x}_{l}\right)+\text { h.o.t. }=0
\end{aligned}
$$

should hold for each $i=1, \ldots L$. In this context, $\boldsymbol{I}$ denotes the equilibrium environment set by the equilibrium of strategies $\boldsymbol{x}^{(1)}, \boldsymbol{x}^{(2)}, \ldots, \boldsymbol{x}^{(L)}$. In the last term of Eq. (7), we replaced $\boldsymbol{I}$ by $\boldsymbol{I}_{\hat{\boldsymbol{x}}}$ because the error caused by the replacement is in the third order. As the adjustable variable $\boldsymbol{I}$ enters the equation through the $K+1$ number of parameters $\alpha(\boldsymbol{I}), \beta_{k}(\boldsymbol{I}), k=1, \ldots, K$, the equilibrium conditions cannot be satisfied generically for $L>K+1$, as it was stated. This bound is related to the principle of competitive exclusion, as the number of locally achievable dimensions of $\boldsymbol{I}$ at $\boldsymbol{I}_{\hat{\boldsymbol{x}}}$ is $K+1$ [15]. (One can change the environmental state from $\boldsymbol{I}_{\hat{\boldsymbol{x}}}$ by changing either the strategy, or the population size, representing together $K+1$ local directions.)

It is clear from the bound that at most $K+1$ branches can appear from one single branching event.

\section{Model}

In line with the general framework presented in the preceeding section, we introduce a specific model for the simulational study. Evolution of a $K=2$ dimensional "strategy" parameter, denoted generally by the vector variables $\boldsymbol{x}, \boldsymbol{y}$, etc., is investigated. The strategy is inherited either faithfully or with a small probability of mutation.

The rate of reproduction $b(\boldsymbol{x})$ of strategy $\boldsymbol{x}$ is specified as

$$
b(\boldsymbol{x})=1-\boldsymbol{x}^{\mathrm{T}} \boldsymbol{A} \boldsymbol{x},
$$

where the matrix $\boldsymbol{A}$ is

$$
\boldsymbol{A}=\left(\begin{array}{cc}
(1-f)^{-1} & 0 \\
0 & 1-f
\end{array}\right)
$$

with the asymmetry parameter $0 \leq f<1$. Accordingly, the central strategy $\boldsymbol{x}=\mathbf{0}$ maximises the reproduction rate. Strategies with $\boldsymbol{x}^{\mathrm{T}} \boldsymbol{A} \boldsymbol{x}>1$ are not viable.

Death is caused by competition with other individuals. Any individual of strategy $\boldsymbol{x}$ contributes to the death rate of an individual of strategy $\boldsymbol{y}$ by $a(\boldsymbol{x}, \boldsymbol{y})$. This "competition function" is specified as

$$
a(\boldsymbol{x}, \boldsymbol{y})=\exp \left(-\frac{(\boldsymbol{x}-\boldsymbol{y})^{2}}{2 \sigma^{2}}\right)
$$

where $\sigma$ is the "competition width". The death rate of an individual of strategy $\boldsymbol{x}$ is determined by the total competition the individual experiences:

$$
I(\boldsymbol{x})=\sum_{j=1}^{L} n^{(j)} \cdot a\left(\boldsymbol{x}^{(j)}, \boldsymbol{x}\right) .
$$


In this model, the function $I(\boldsymbol{x})$ plays the role of the environmental interaction variable $\boldsymbol{I}$. That is, $\operatorname{dim} \boldsymbol{I}=\infty$ and there is no trivial bound on the number of coexisting strategies.

Suppose, that the strategies $\boldsymbol{x}^{(i)}, i=1,2, \ldots, L$ are present with population sizes $n^{(i)}$. If all the $n^{(i)}$ s are large enough and there are no mutations, the population dynamics can be described by the Lotka-Voltera competition equations

$$
\frac{d n^{(i)}}{d t}=\left[b\left(\boldsymbol{x}^{(i)}\right)-\boldsymbol{I}\left(\boldsymbol{x}^{(i)}\right)\right] \cdot n^{(i)}
$$

The growth rate

$$
r(\boldsymbol{y}, \boldsymbol{I})=b(\boldsymbol{y})-I(\boldsymbol{y})
$$

is considered to be the "fitness" of the strategy $\boldsymbol{y}$ in the background set by the strategies $\boldsymbol{x}^{(j)}$ and population sizes $n^{(j)}, j=1,2, \ldots, L$.

The mutation process has to be implemented by hand. We suppose that strategy $\boldsymbol{x}^{(i)}$ mutates with rate $\mu b\left(\boldsymbol{x}^{(i)}\right) n^{(i)}$. (It corresponds to probability $\mu$ of mutation in any birth event $-\mu$ is often called mutation rate) The new strategy $\boldsymbol{x}^{(\text {mut })}$ is chosen randomly with uniform distribution in the two-dimensional $\varepsilon$ neighbourhood of $\boldsymbol{x}^{(i)}$. While the size of the mutant clone is small compared to the "resident" densities $n^{(i)}, i=1,2, \ldots, L$, the death rate of a mutant individual is dominated by the contributions from individuals of the resident strategies. Then, the growth rate of the mutant can be calculated from Eq. (13). However, while the mutant population is small, the deterministic population dynamics does not apply. According to the branching process theory, long-term survival probability of the mutant clone, descending from a single individual, is

$$
P=\left\{\begin{array}{cll}
0 & , \text { if } & r \leq 0 \\
r / b & , \text { if } & r>0
\end{array}\right.
$$

[37, p. 109][41, 42]. The new mutant is considered to be established, and included into the list of the strategies present, with the probability (14) calculated for $r=r\left(\boldsymbol{x}^{(\mathrm{mut})}\right)$. The newly established strategy begins its life from a low initial population size $n^{\text {(mut) }}$.

Strategies decreasing below a given population size $n_{\text {ext }}$ are removed from the list of strategies.

Accordingly, the simulation of the model consists of repeating 3 consecutive steps:

- integrating the ODE of the population dynamics for a period $\tau$;

- removal of the strategies with low population size;

- possible addition of new mutant(s).

This combination of deterministic population dynamics with a stochastic mutation process was introduced by Metz et al. [15] and Geritz et al. [17] and, since then, applied for several models [43, 44, 45, 46, 47, 48].

The mutation rate was chosen to be $\mu=2 \cdot 10^{-7}$. The update time was $\tau=100$ time unit, small enough to keep the expected number of mutations during $\tau$ below 1 . The density of the arising mutant and the extinction threshold were equal and small enough that the arising mutant does not disturb the resident: $n^{\text {(mut) }}=n_{\text {ext }}=10^{-6}$. The resolution of the strategy space was rather fine: $4 \cdot 10^{-6}$ unit. 


\section{Results}

\subsection{The pattern of phase transitions to evolutionary branching}

Note first that if the strategy $\boldsymbol{x}$ alone is present, then the only singular strategy is $\boldsymbol{x}=0$. The equilibrium density (as determined by the condition $r=0$ ) for this strategy when alone, is $n=1$. Then, for $f=0$, the fitness function in this equilibrium is

$$
r(\boldsymbol{y}, \boldsymbol{I})=1-\boldsymbol{y}^{2}-\exp \left(-\frac{\boldsymbol{y}^{2}}{2 \sigma^{2}}\right) .
$$

At $\boldsymbol{y}=0$ it has a local maximum for $\sigma>\sigma_{\mathrm{c}}$, and a local minimum for $\sigma<\sigma_{\mathrm{c}}$, where $\sigma_{\mathrm{c}}=1 / \sqrt{2}=0.707$. This threshold is independent of the number of dimensions.

Figs. 1-4 demonstrate the behaviour of the model with competition widths $\sigma=$ $1.0,0.7,0.5,0.2$, respectively, in $2 \mathrm{D}$ for the rotationally symmetric $(f=0)$ case. In the bottom left part of each figure the trajectory in the strategy space is shown, while in the top left and bottom right part of the figures the time development of each component of the strategies is shown. (We will use the same representations in the rest of the paper.) The simulation was initiated with one species of strategy of $\boldsymbol{x}^{(0)}(t=0)=(0.1,0.1)$, the mutational step-size was $\varepsilon=0.005$.

In all cases, evolution of a single species converges to $x=0$. For $\sigma>\sigma_{\mathrm{c}}$ this strategy is an ESS, so evolution ceases here. On the other hand, $\sigma<\sigma_{\mathrm{c}}$ results in evolutionary branching. This change of the behaviour of the singular point at $\sigma=\sigma_{\mathrm{c}}$ is analogous to a phase transition with the competition width $\sigma$ as control parameter. The rate of the branches' expansion can be regarded as the order parameter of the phase transition. Random establishment of the branching direction represents a spontaneous breaking of the rotational symmetry of the model.

The global behaviour is in good agreement with the theoretical predictions. After the first branching the evolution of the emerging species are directional again until they arrive to the vicinity of their respective singular points. These new singularities are determined by the condition that the evolutionary attraction towards to the maximum of carrying capacity should compensate for the repulsion between the species due to competition. The new singularities may or may not be new branching points depending on the value of the control parameter $\sigma$. So, further decrease of the control parameter results in consecutive branching transitions. For extremely small $\sigma$ values a whole series of branchings occurs (see Fig. 4). However, even in the case of small $\sigma$, when the propensity for branching is very high, we have never found any branching into more than three directions, in accordance with the analytic prediction.

Nevertheless, a remarkable departure from the analytic theory is also observable for small competition widths $(\sigma=0.2,0.5)$. The first branching may occur before reaching the singular strategy $\boldsymbol{x}=0$. This is because the analytic treatment supposes the validity of the linear approximation for the fitness function on the scale of mutational step-size $\varepsilon$. This assumption breaks down at the singular point, where the linear term diminishes. For very small $\sigma$, the quadratic term arising from the competition function (10) is large enough to dominate the fitness function earlier than the singular strategy is approached.

\subsection{The branching pattern}

The parameter value $\sigma=0.5$ was chosen for more detailed investigations of the branching pattern. In this case the first branching goes already rather easily but the number of consecutive branchings is still limited. Consequently, the system evolves deterministically 

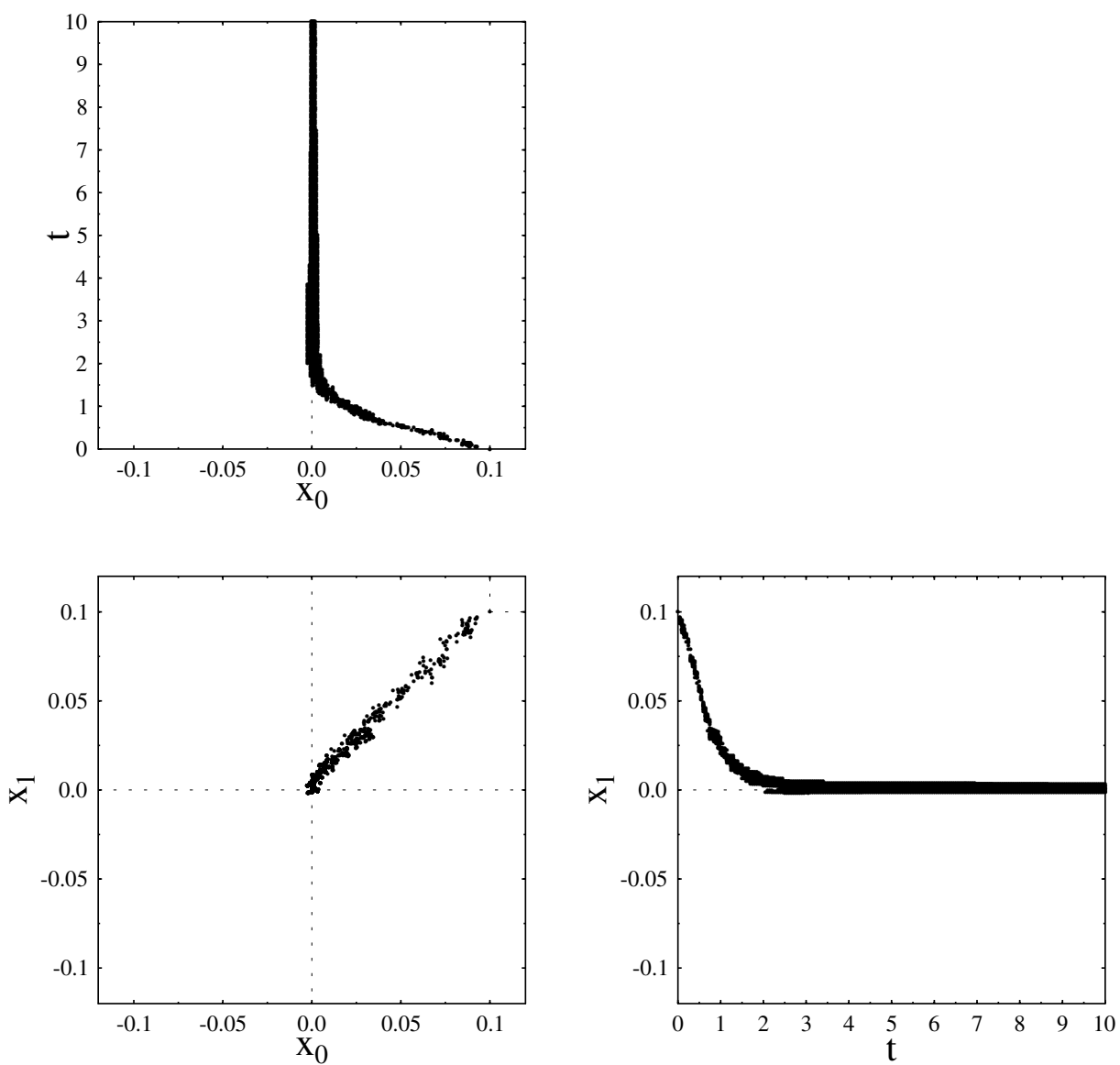

Figure 1: Evolution with competition width $\sigma=1.0$. The system starts from $(0.1,0.1)$ and evolves into the singular point $(0,0)$. There is no branching because $\sigma>\sigma_{c}$. The carrying capacity is symmetric: $f=0$; the mutational step-size is $\varepsilon=0.005$. In the bottom-left part of the Figure the strategy-space trajectory is shown, while in the topleft and bottom-right parts the development of the strategy components $x_{0}$ and $x_{1}$ are depicted, respectively. Time is counted by million time units. 

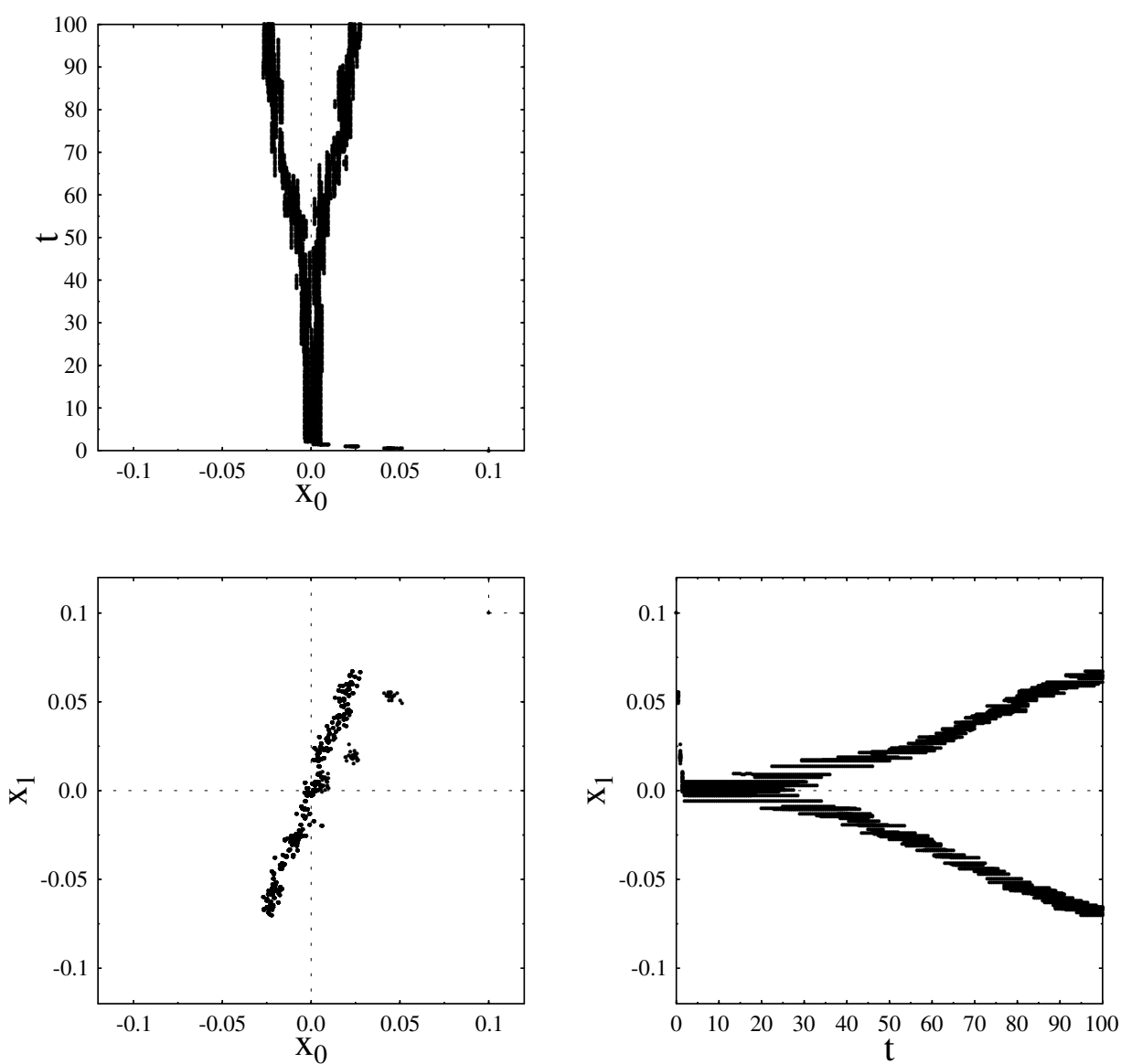

Figure 2: Same as Fig. 1, but the competition width $\sigma=0.7$, slightly less than $\sigma_{c}$. There is already a branching, but it goes rather slowly: it occurs at about 20 million TU. 

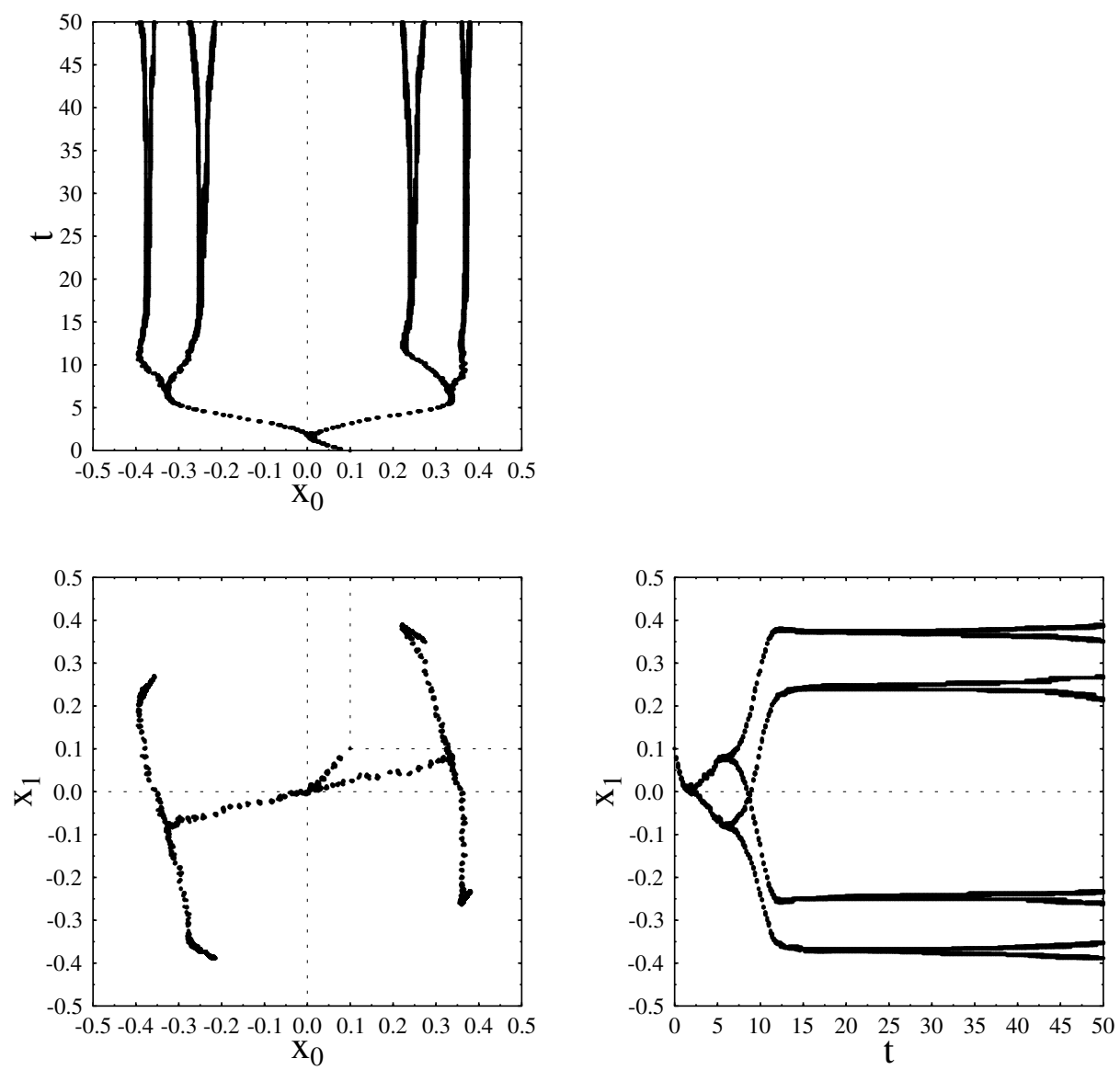

Figure 3: Same as Fig. 1, but $\sigma=0.5$. The branching occurs more easily than in Fig. 2, at about 2.5 million TU. 

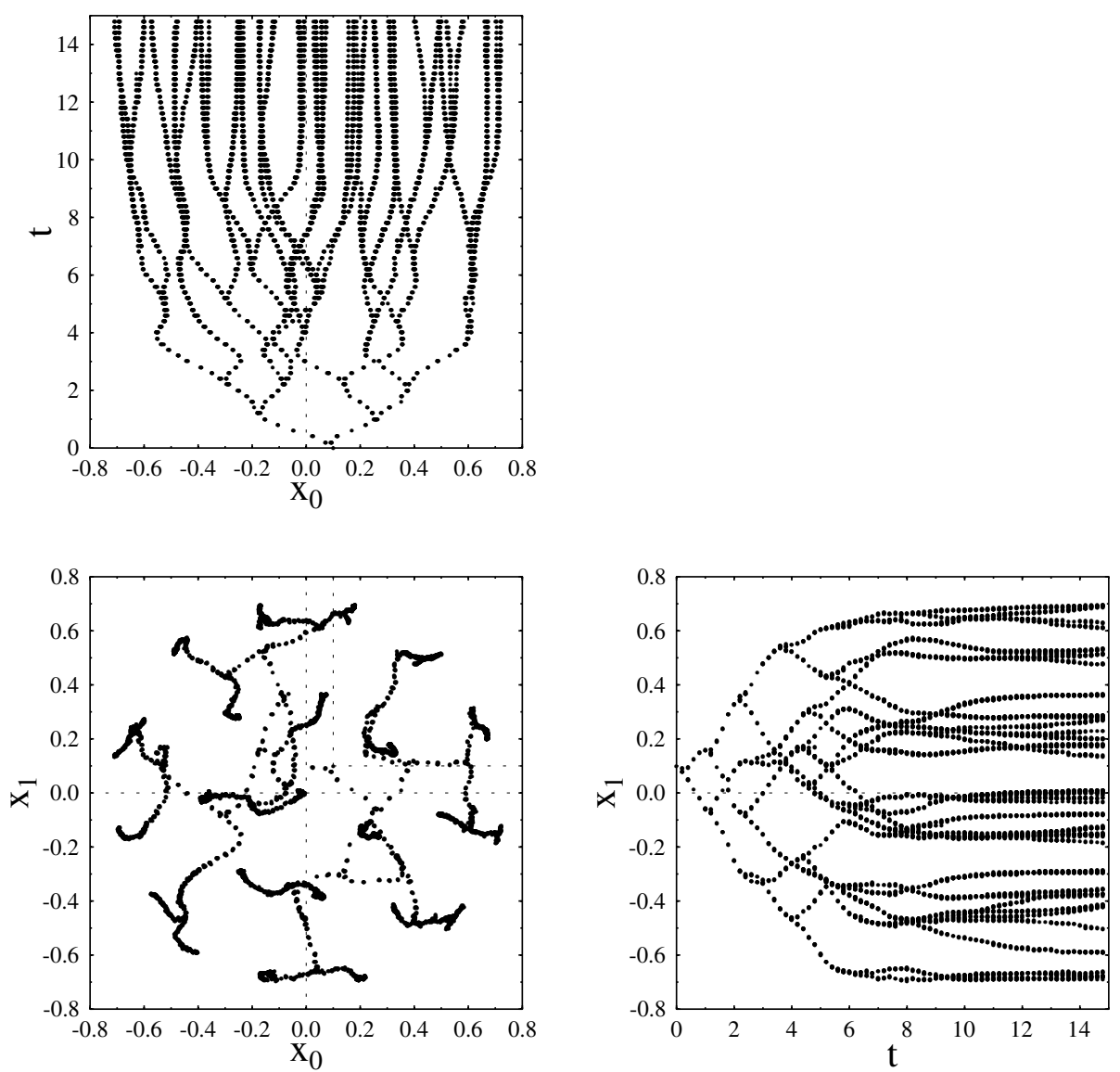

Figure 4: Same as Fig. 1, but with very small competition width $(\sigma=0.2)$. A series of consecutive branchings occurs. 
for a sufficiently long time after each branching event. In this Section, we concentrate on the first branching at $\boldsymbol{x}=0$. Accordingly, the simulations are initiated by a single species with this strategy.

Some examples are shown in Fig. 5 with branchings into two directions. For the sake of illustration in Fig. 6 we show examples where the branching at the centre occurs into three directions, but during further evolution one of the branches dies out. These are not regarded as three-branchings when studying statistics. In Fig. 7 "real" three-branchings can be seen. In these figures the mutational step-size is $\varepsilon=0.0025$. Some other examples with mutational step-size $\varepsilon=0.005$ for two-branchings are presented in Fig. 8, for threebranchings in Fig. 9. We conclude that both the two- and the three-branchings are really existing phenomena.

The possibility of consecutive branchings strongly depends on the outcome of the first branching, whether it happened into two or three directions. After a two-branching event in the centre, both branches separate into two by a new branching event on a circle of diameter 0.7. Then, the four branches evolve into another branching point where all the four branches separate into two, again. The eight-branches system evolves until it reaches a roughly symmetric configuration when all the eight branches are situated on a circle of diameter 0.875 . This is the final steady stage of the evolutionary process (Fig. 10). In contrast, the three branches emerging from a three-branching event branch only once more. These branching points are situated on a circle of diameter 0.85. The six branches emerging here evolve onto approximately the same circle as in the two-branching case, and ceases to evolve at the symmetric configuration (Fig. 11).

In the upper-right part of Figs. 10, 11 we have depicted the time evolution of the number of coexisting phenotypes $L$ and the density of the whole population $n=\sum_{j=1}^{L} n^{(j)}$. As we see, the density of the whole population increases after every branching. The number of the coexisting phenotypes $L$ becomes rather stochastic, but tends to increase near the branching points. It decreases again when the system leaves a branching point. The reason for this behaviour is the flattening fitness function around the singularities. At these regions, the (stochastic) process of mutations dominates over selection caused by the slope of the fitness landscape.

Finally, Fig. 12 with $\varepsilon=0.01$ and $f=0.2$ demonstrates the consequences of strong breaking of rotational symmetry. In this case the final stationary state consists of branches situated on an ellipse. The asymmetry makes the three-branchings rather improbable. For example, with the parameter set of these examples, we have found only 11 three-branchings on 3000 .

\subsection{Statistics of the branching types}

In this section we measure the probability of three-branchings at several parameter combinations, but always with $\sigma=0.5$. We are most interested to see whether this probability goes to zero, or remains finite, in the limit $\varepsilon \rightarrow 0$.

At the end of each simulation, a clustering algorithm was used to determine whether a two- or a three-branching had occurred. The two strategies nearest to each other were fused in each step of the algorithm. Doing so, the minimal distance between the phenotypes increased step by step slowly while both phenotypes to be fused were in the same branch. However, it had a big jump when, finally, strategies from two different branches were tried to be fused. The algorithm was terminated when the minimal distance reached the value 0.2 . Then, the still different phenotypes were counted and the number was regarded indicative of the number of branches. As we were interested in the number of branches reaching the deterministic stage of diverging evolution, the clustering had to be performed 

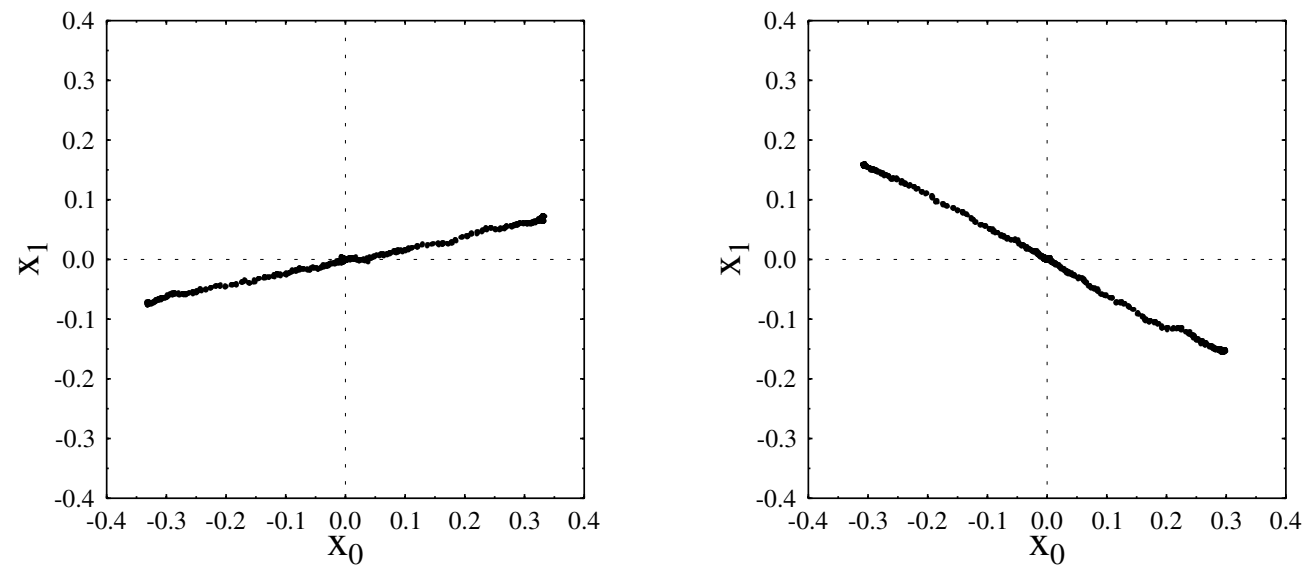

Figure 5: Examples for two-branchings with $\sigma=0.5$ in the symmetric situation $(f=0)$, with $\varepsilon=0.0025$, which is a rather small value. The system starts from the centre.
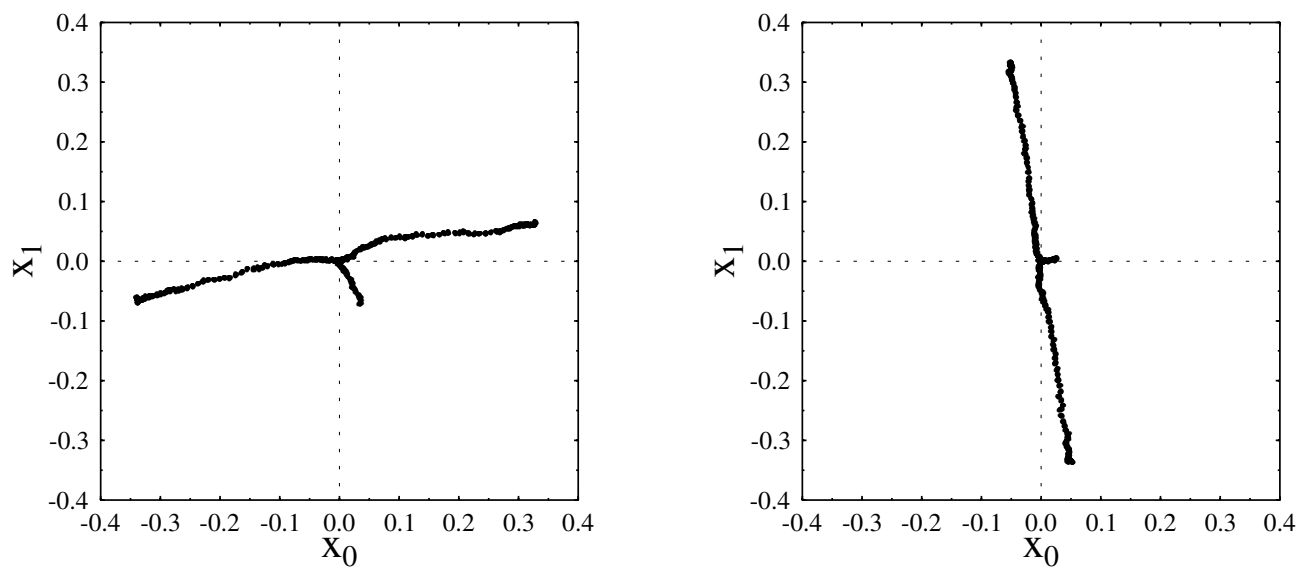

Figure 6: Examples with the same parameters as in Fig. 5, for the extinction of one of the three branches before long after the branching in the centre. Development like these is not considered as a three-branching when investigating statistics. 

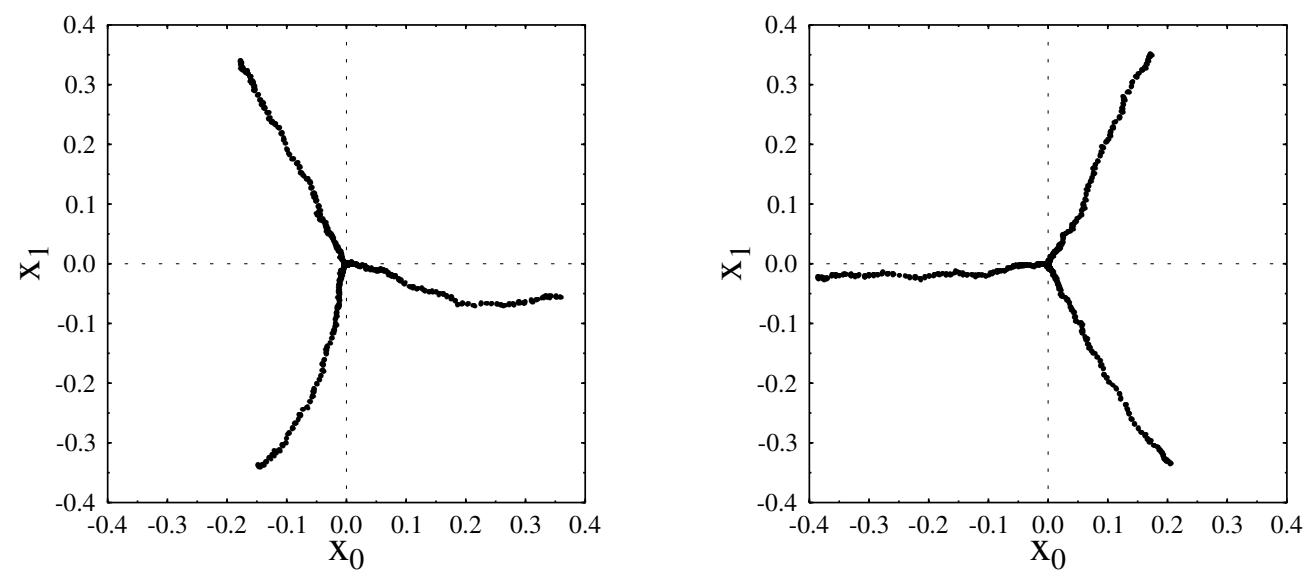

Figure 7: Real three-branchings with the same parameters as in Fig. 5.
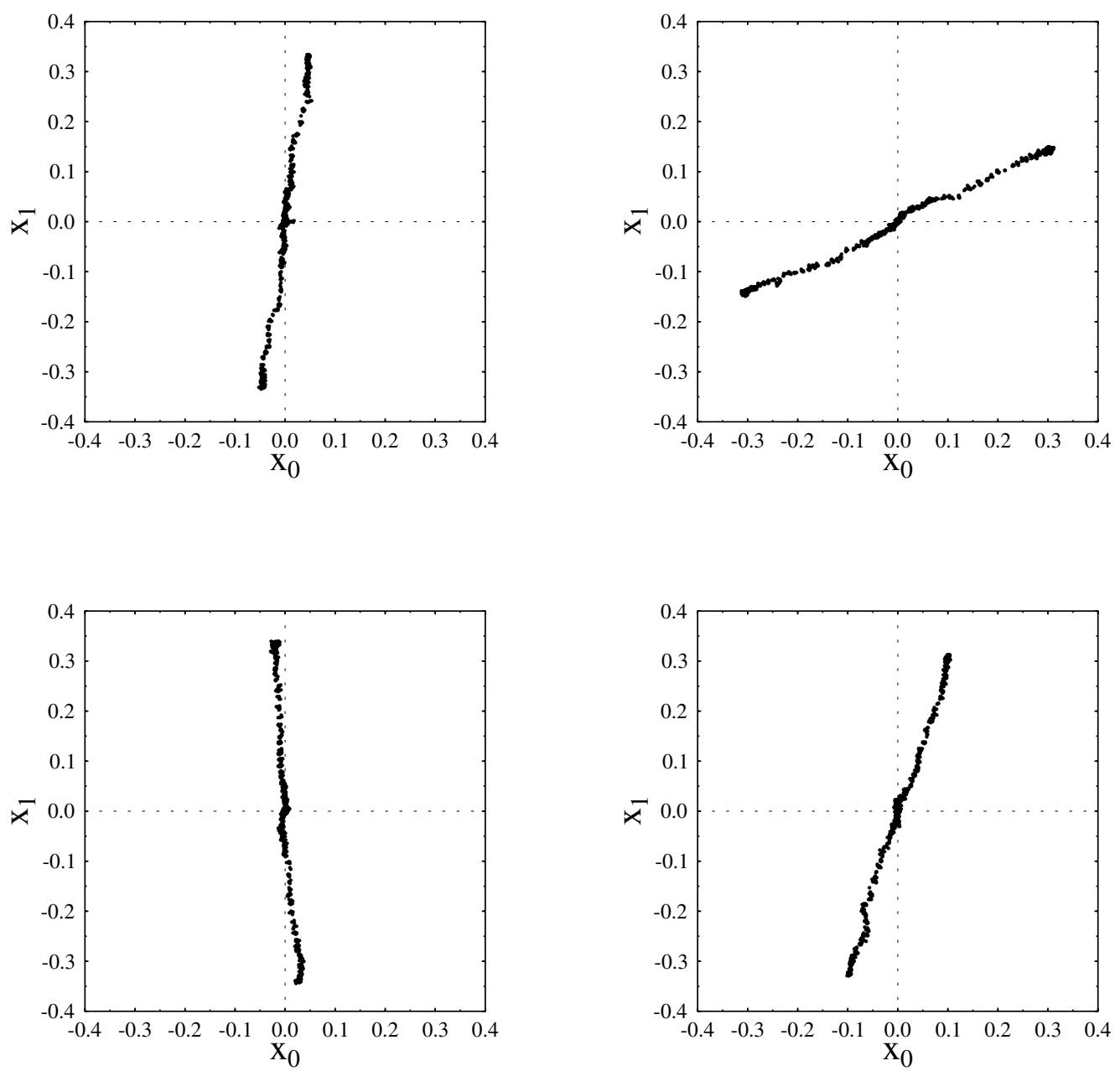

Figure 8: Examples for two-branchings with the same parameters as in Fig. 5, but with larger mutational step-size $\varepsilon=0.005$. 

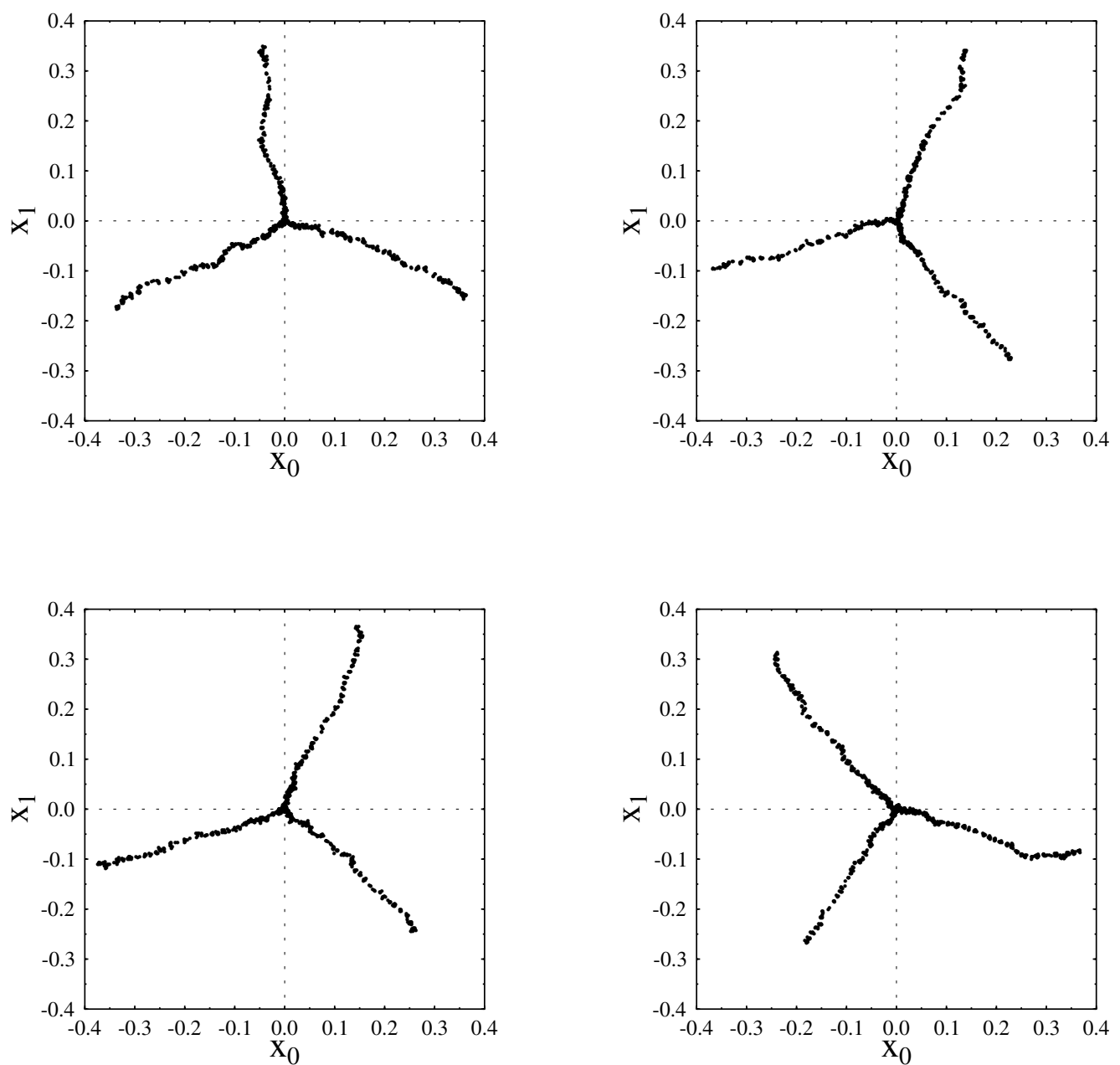

Figure 9: Examples for three branchings with the same parameters as in Fig. 8. 

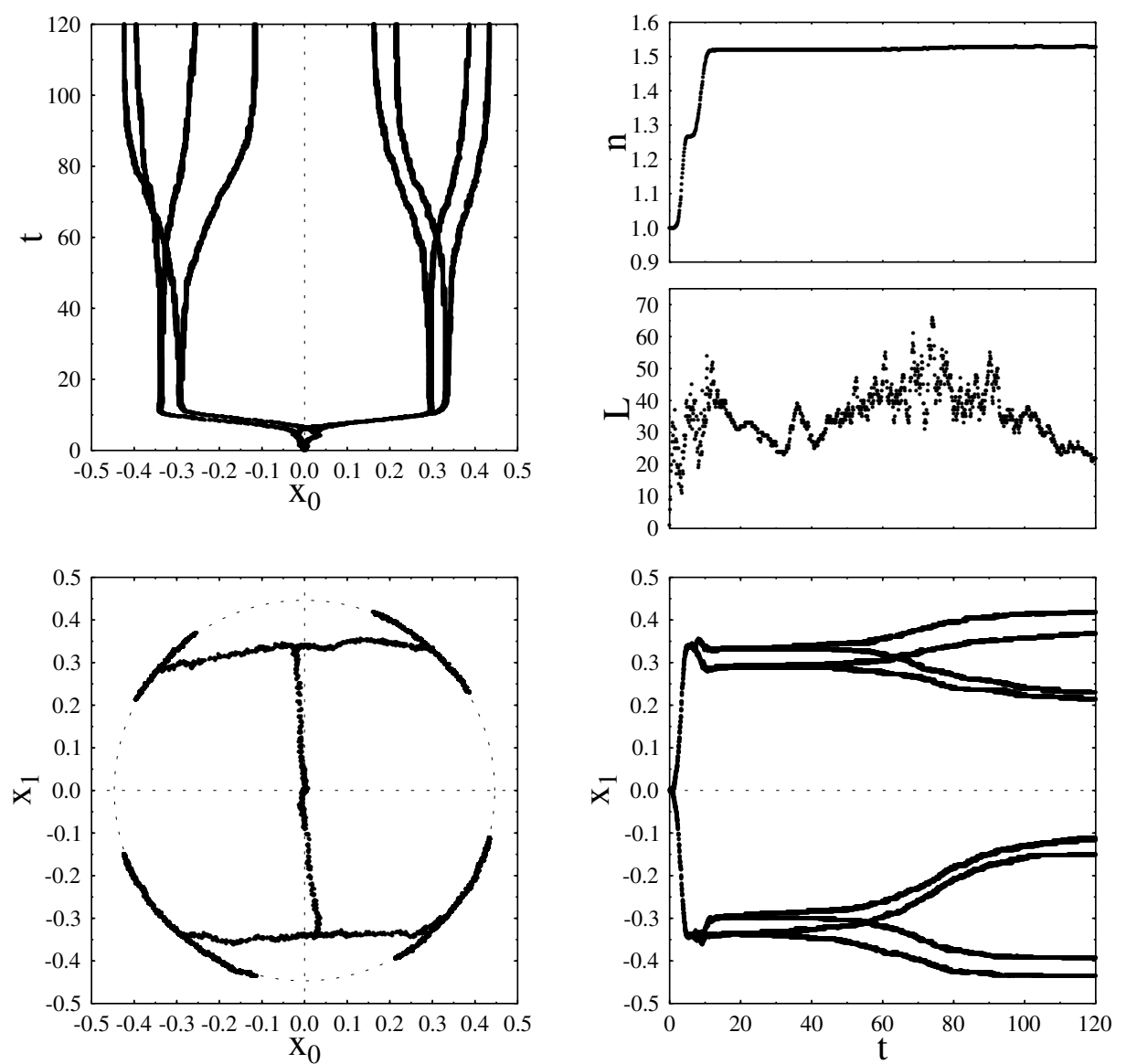

Figure 10: The same parameters as in Fig. 8, but the system has been let evolve for a long time to reach a steady state state. The steady state consists of eight branches situated on a circle in the strategy space. The trajectory and time developments are also shown, and in the upper-right part we have depicted the time development of the number of coexisting phenotypes $L$ as well as the density of the whole population $n=\sum_{j=1}^{L} n^{(j)}$. The time scale of these small figures is the same as the one of the bottom-right figure. 

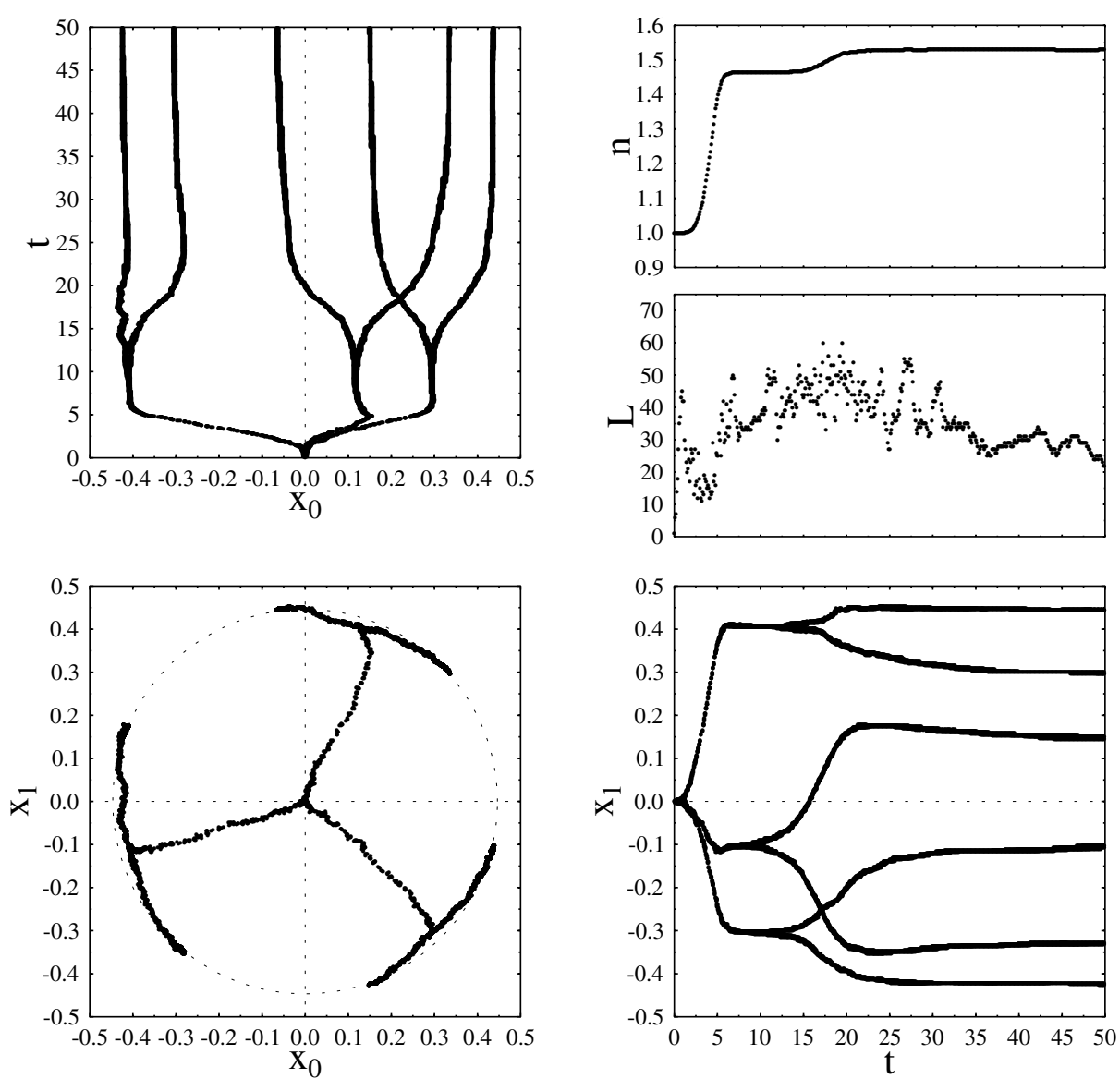

Figure 11: Similar to Fig. 10, but the first branching occurred into three directions. In this case the steady state consists of six branches situated on the same circle in the strategy space. 

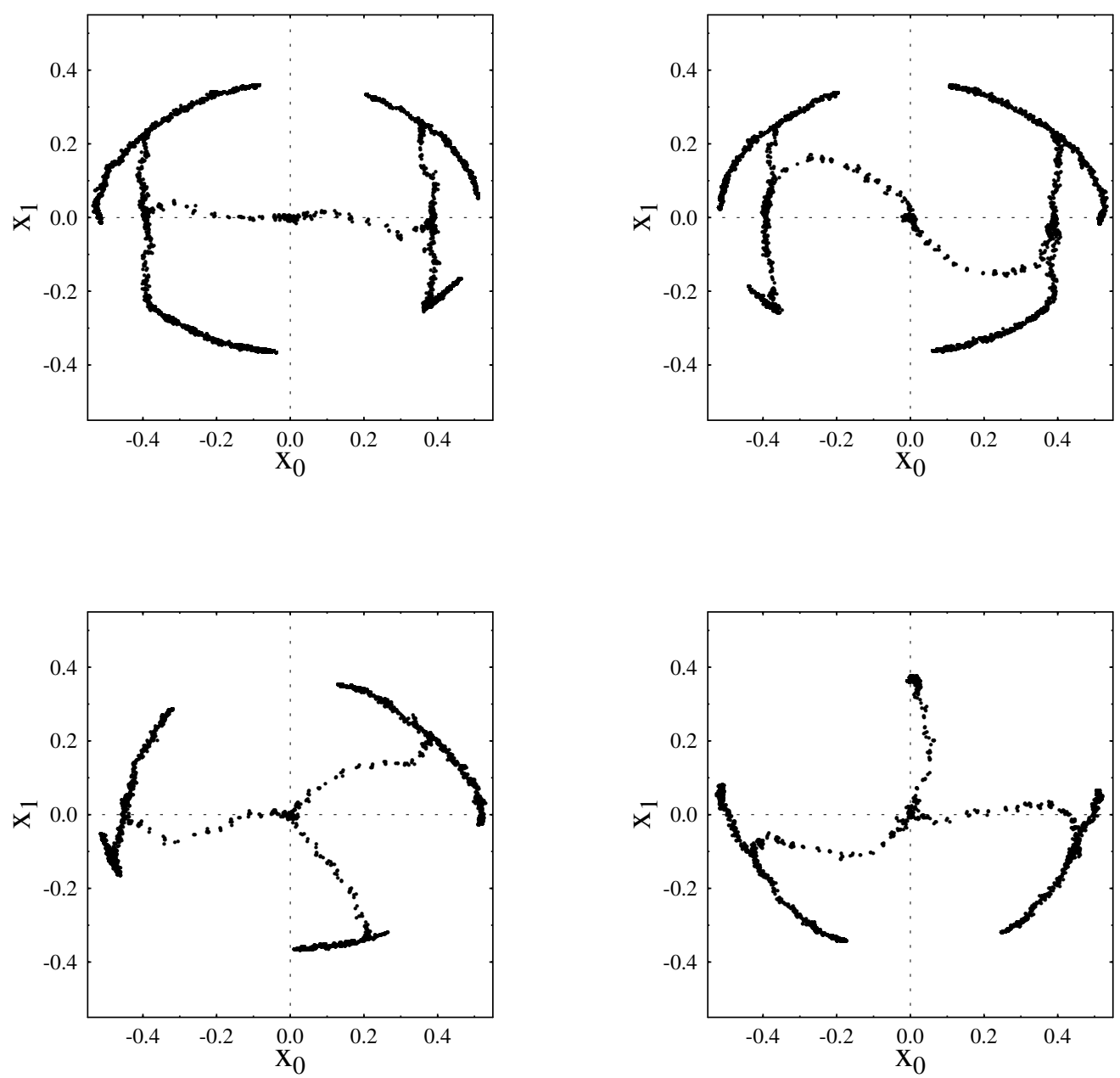

Figure 12: Two- and three-branchings in a highly asymmetric situation with mutational step-sizes $\varepsilon=0.01$ and asymmetry parameter $f=0.2$. The final steady situations are shown with the branches situated on an ellipse. 


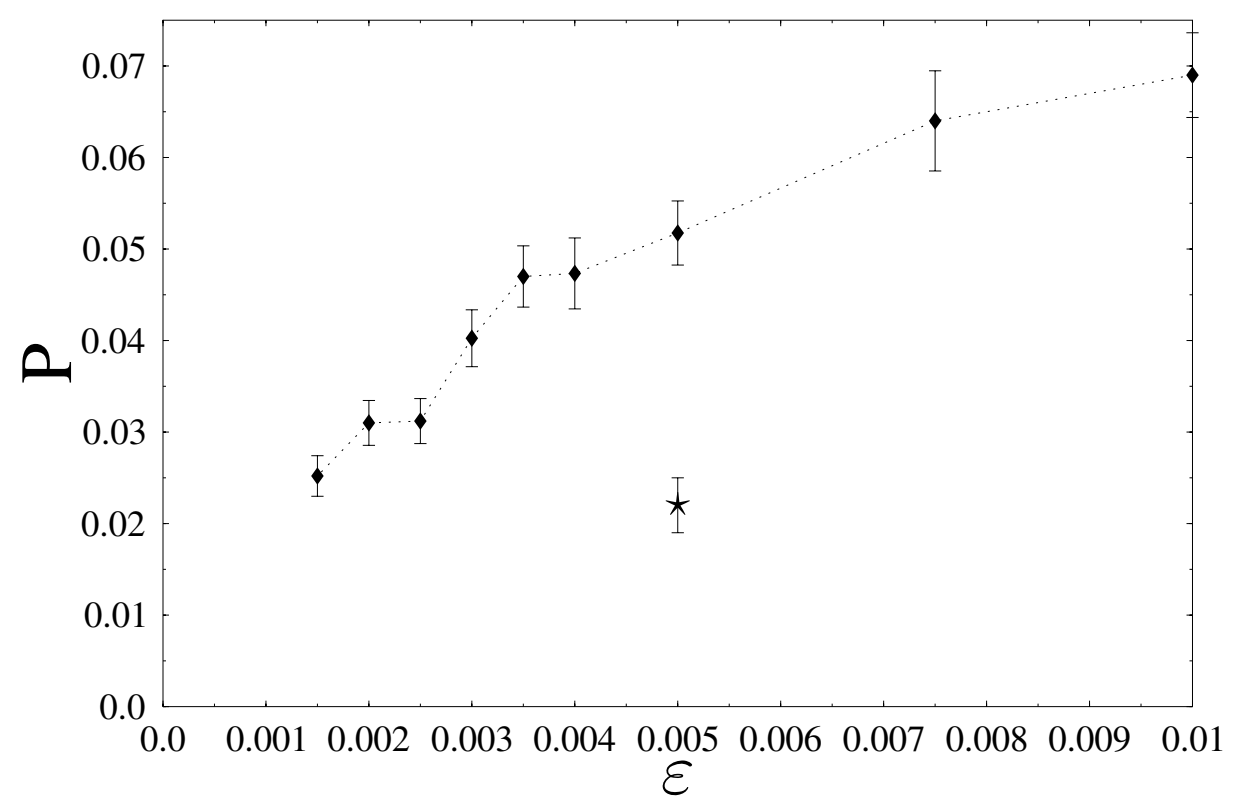

Figure 13: Probability of three-branchings as a function of $\varepsilon$. The carrying capacity is rotationally symmetric $(f=0)$. The single point marked by star indicates the situation where the starting point was $\boldsymbol{x}^{(0)}(t=0)=(0.1,0.1)$, instead of the singular one.

far enough from the branching points. Accordingly, clustering was carried out when the separation of the branches had reached the distance 0.48. (Obviously, this distance should be larger than the critical minimal distance 0.2 of the clustering algorithm.)

In Fig. 13 we have depicted the dependence of the probability of three-branchings as a function of the mutational step-size $\varepsilon$ for the rotationally symmetric $(f=0)$ case. Each point in the figure represents 2000-5000 simulations to achieve the precision indicated by the error bars. (See Tab. 1 for the time needed to reach the separation 0.48.)

In Fig. 13 we see that the smaller the mutational step-size is, the smaller the probability of three branchings we have. For a three-branching, it is necessary that the three mutants take a rather symmetric position around the centre to be able to live together. When the mutational step-size is large, the mutants are more "mobile" in the strategy space: there is more possibility to correct the eventual defects of the starting position. Numerical studies become especially difficult for very small values of $\varepsilon$ for two reasons. First, because simulation of evolution becomes slow and, second, because very small probabilities are to be measured in this case. Still, extrapolation for $\varepsilon \rightarrow 0$ seems to indicate vanishing ratio of three-branchings.

The three-branching probability as a function of the asymmetry parameter $f$ is presented in Fig. 14 for two different mutational step-sizes, $\varepsilon=0.01$ and $\varepsilon=0.005$. Increasing asymmetry makes the three-branchings less probable. This is easily understood if we consider that increasing the asymmetry signifies out a specific direction for branching.

Both Fig. 13 and Fig. 14 contain one single data point marked by a star, representing a simulation in which the starting point was $\boldsymbol{x}^{(0)}(t=0)=(0.1,0.1)$, instead of the singular one. Starting the population out of the singular point makes the tree-branchings less 


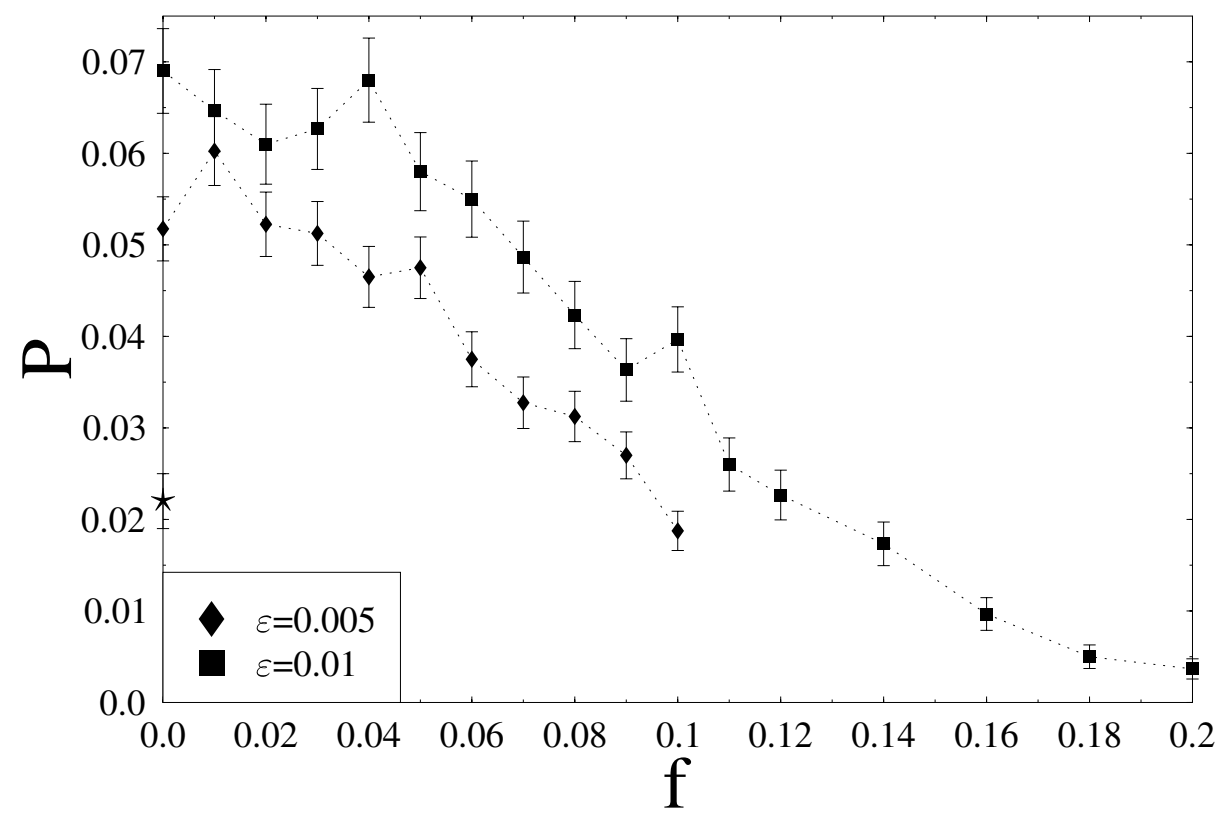

Figure 14: Probability of three-branchings as a function of the asymmetry factor $f$ at two different values of the mutational step-size $\varepsilon$. The probability vanishes with increasing $f$. The single point marked by star indicates the situation where the starting point was $\boldsymbol{x}^{(0)}(t=0)=(0.1,0.1)$, instead of the singular one.

Table 1: Time that the branches need to reach the separation 0.48 as a function of the step-size $\varepsilon$

\begin{tabular}{|cc|}
\hline$\varepsilon$ & million TU \\
\hline 0.0015 & 40 \\
0.002 & 20 \\
0.0025 & 15 \\
0.003 & 9 \\
0.0035 & 7 \\
0.004 & 5.5 \\
0.005 & 3.5 \\
0.0075 & 2 \\
0.01 & 1 \\
\hline
\end{tabular}


probable since it also corresponds to a breaking of the rotational symmetry.

\section{Conclusions}

In line with the theoretical expectations [28], our simulations demonstrated that a series of small and rare random mutation steps results in a deterministic process of continuous evolution, governed by the slope of the fitness function, whenever this slope is non-zero. On the other hand, the behaviour remains of stochastic nature in the vicinity of singular strategies, where the fitness gradient vanishes. Here, the selection is weak, so the number of concurrent strategies remains high even at low mutation rate. The region of essential randomness can be shrunk arbitrarily by choosing the mutational step-size $\varepsilon$ small enough. However, the deterministic evolution will drive the system into the stochastic region in any case, provided that the singular point in question is convergence stable. If evolutionary branching occurs at a non-ESS singular point, both the number and the directions of the emerging evolutionary branches are essentially random as they are determined by the interface between the stochastic and the deterministic phase. We observed branchings into two and three directions in 2D strategy space in line with the theoretical bound on the number of emerging branches.

The results of our simulations point to vanishing three-branching probability in the limit $\varepsilon \rightarrow 0$. Care is needed to compare this finding with the deterministic limit away from the singularity, which is also related to $\varepsilon \rightarrow 0$. Decreasing $\varepsilon$ makes evolution slower, so it rescales time. This rescaling goes as $\varepsilon^{-1}$ in deterministic phase, when the fitness difference is dominated by the linear term, and as $\varepsilon^{-2}$ in the vicinity of the singularity under the dominance of the quadratic term. Consequently, time scales of the directional evolution and of the branching separate in the limit $\varepsilon \rightarrow 0$. We loose branching on the fast scale and directional evolution becomes instantaneous on the slow one. (See [21] for the connection between this time-scaling issue and the concept of punctuated equilibrium [49].) Our simulations corresponded to an intermediate $\varepsilon$, for which the directional evolution was already deterministic enough, but the time-scale separation was not extreme and the threeway branching phenomenon was still present. It is a question for further consideration, what possible choice of $\varepsilon$ corresponds the best to the biological reality. Considering sexual populations, in which recombination maintains a high level of genetic variance even in the limit of small and rare mutations, may even decouple these two consequences of small evolutionary steps.

Breaking of rotational symmetry of the model also decreases the probability of threebranchings. The relative scales of the two directions were fixed when the mutation covariance matrix $\boldsymbol{C}$ was chosen to be rotationally symmetric. Without the freedom of further rescaling, in reality we have little reason to expect rotationally symmetric $\boldsymbol{A}$ matrix corresponding to $f=0$. Breaking the rotational symmetry of the competition function (10) would have a similar consequence. This result, again, points to the probable predominance of the two-way branchings.

Furthermore, we have found that the initial condition $\boldsymbol{x}^{(0)}(t=0) \neq 0$ results in a smaller probability of three-way branching as well, than the initial condition $\boldsymbol{x}^{(0)}(t=$ $0)=0$ does. Naively, one could assume that the deterministic convergence to the singular point diminishes the effect of the initial condition on the outcome of branching. According to our data, this is certainly not the case. Once again, the intimate relation between the deterministic and the stochastic phase of the process provides the explanation. The convergence ceases as soon as the system enters the stochastic region, so the stochastic phase is initiated by a rotationally asymmetric distribution. These two types of initial 
conditions represent different biological scenarios, both of them are realistic. The initial strategy $\boldsymbol{x}^{(0)}(t=0) \neq 0$ corresponds to a speciation process in an essentially constant environment. One species evolves to the branching singularity and splits there. On the other hand, the initial condition $\boldsymbol{x}^{(0)}(t=0)=0$ is relevant when speciation is initiated by an environmental change. Suppose, that a parameter change bifurcates a former ESS point into a branching one. If a species has evolved earlier into this singularity then it starts the process of branching from an already established rotationally symmetric distribution after the bifurcation.

All these results together suggest that the three-way branching is possible, still, the dominant mode of speciation is probably the two-way branching. This is certainly in line with the usual picture about the evolutionary process. However, no clear empirical way is available to decide whether each new species emerging during adaptive radiation comes from a separate event of branching. While the number of trait dimensions is high in any real evolutionary process, our results support the possibility that only one combination of them is relevant in any specific event.

The Lotka-Voltera competition model we analysed, is the most common model of coexistence of different species, as well as of different genotypes within the same species [36, $50,51,52,53,54]$ and one of the simplest example for evolutionary branching. However, as far as relatively small mutation steps are considered, our results are independent of the specific model we studied. This is clear from the fact that, for small $\varepsilon$, the process of mutant invasion is determined by the first non-vanishing term of the Taylor expansion of the fitness function. Directional evolution is determined by the slope, while the stochastic phase in the vicinity of the singularity is governed by the second order terms. This is exactly the idea behind the classification of the singular strategies according to their second-order behaviour, which was suggested and implemented in [16] for 1D strategy space. Note, that the mutation rate $\mu$ was not low enough to strictly obey the conditions of the analytic theory. The next mutant arrived well before the previous one has equilibrated. However, according to the simulation results, this does not affect the behaviour too much. In line with Eigen's concept of quasi-species $[55,56,57]$, the directional part of the evolutionary process can be described as a moving cloud of mutants.

The only essential assumption we rely on is the concept of adaptive speciation driven by an ever-changing fitness landscape. For a complete theory of speciation, one has to consider a sexual population and implement a mechanism for emergence of reproductive isolation between the species $[3,5,7,10,12]$. Evolutionary studies often employ a fixed fitness function. This approach is very useful in studying evolutionary optimality problems, as well as mutation-selection balance $[58,59,60,61]$. However, no meaningful biological diversity can emerge in such model, as a consequence of adaptation, because there is no generic reason for the different peaks of the adaptive landscape to have of equal height.

In contrast with adaptive speciation, the classical "allopatric" theory of speciation $[62,63]$ supposes that separation between species emerge as a genetic "by product" [64] of evolution of the different sub-populations at different locations. There is no explicit need for changing fitness landscape in this picture. Implicitly, however, the assumption of feedback is needed to understand why the new and the old species can live together, without out-competing each other, when they finally meet. New empirical evidence support that spatial separation is not a prerequisite for speciation $[65,66]$. On the other hand, the idea of adaptive speciation might be applicable for speciation modes involving spatial segregation $[8,9,44]$, so it has a chance of becoming the general underlying concept of speciation. 


\section{References}

[1] Rosenzweig, M. L. (1978) Competititve speciation. Biological Journal of the Linnean Society 10, 275-289.

[2] Rosenzweig, M. L. (1995) Species diversity in space and time. Cambridge University Press, Cambridge.

[3] Seger, J. (1985) Intraspecific resource competition as a cause of sympatric spciation. In Evolution. Essays in honour of John Maynard-Smith, eds. Greenwood, P., Harvey, P., \& Slatkin, M., Cambridge University Press, Cambridge.

[4] Doebeli, M. (1996) A quantitative genetic competition model for sympatric speciation. J. Evol. Biol. 400, 354-357.

[5] Dieckmann, U. \& Doebeli, M. (1999) On the origin of species by sympatric speciation. Nature 400, 354-357.

[6] Doebeli, M. \& Dieckmann, U. (2000) Evolutionary branching and sympatric speciation caused by different types of ecological interactions. The American Naturalist 156, S77-S101.

[7] Geritz, S. A. H. \& Kisdi, É. (2000) Adaptive dynamics in diploid sexual populations and the evolution of reproductive isolation. Proc. R. Soc. Lond. B. 267, 1671-1678.

[8] Mizera, F. \& Meszéna, G. (2003) Spatial niche packing, character displacement and adaptive speciation in an environmental gradient. Evolutionary Ecology Research 5, $1-20$.

[9] Doebeli, M. \& Dieckmann, U. (2003) Speciation along environmental gradients. Nature 421, 259-264.

[10] Drossel, B. \& McKane, A. (2000) Competitive speciation in quantitative genetic models. Journal of Theoretical Biology 204, 467-478.

[11] van Doorn, Sander, G., \& Weissing, F. J. (2001) Ecological versus selection models of sympatric speciation: a synthesis. Selection 2, 17-40.

[12] Matessi, C., Gimelfarb, A., \& Gavrilets, S. (2001) Long term buildup of reproductive isolation promoted by disruptive selection: how far does it go? Selection 2, 41-64.

[13] Dieckmann, U., Metz, J. A. J., Doebeli, M., \& Tautz, D., eds. (in press) Adaptive Speciation. Cambridge University Press, Cambridge.

[14] Darwin, C. (1859) On the origin of species by means of natural selection, or the preservation of favoured races in the struggle for life. Harward University Press.

[15] Metz, J. A. J., Geritz, S. A. H., Meszéna, G., Jacobs, F. J. A., \& van Heerwaarden, J. (1996) Adaptive dynamics, a geometrical study of the consequences of nearly faithful reproduction. In Stochastic and spatial structures of dynamical systems, eds. van Strien, S. \& Lunel, S. V., North Holland, pp. 183-231.

[16] Geritz, S. A. H., Metz, J. A. J., Kisdi, É., \& Meszéna, G. (1997) The dynamics of adaptation and evolutionary branching. Physical Review Letters 78(10), 2024-2027. 
[17] Geritz, S. A. H., Kisdi, É., Meszéna, G., \& Metz, J. A. J. (1998) Evolutionary singular strategies and the adaptive growth and branching of evolutionary trees. Evolutionary Ecology 12, 35-57.

[18] Brown, J. S. \& Vincent, T. L. (1987) Coevolution as an evolutionary game. Evolution 41, 66-79.

[19] Brown, J. S. \& Vincent, T. L. (1992) Organization of predator-prey communities as an evolutionary game. Evolution 46, 1269-1283.

[20] Vincent, T. L., Cohen, Y., \& Brown, J. S. (1993) Evolution via strategy dynamics. Theoretical Population Biology 44, 149-176.

[21] Geritz, S. A. H., Kisdi, É., Meszéna, G., \& Metz, J. A. J. (in press) Adaptive dynamics of speciation. In Adaptive Speciation, eds. Dieckmann, U., Metz, J. A. J., Doebeli, M., \& Tautz, D., Cambridge University Press, Cambridge.

[22] Eshel, I. (1983) Evolutionary and continuous stability. Journal of theoretical Biology 103, 99-111.

[23] Taylor, P. (1989) Evolutionary stability in one-parameter models under weak selection. Theoretical Population Biology 36, 125-143.

[24] Christiansen, F. B. (1991) On conditions for evolutionary stability for a continuously varying character. Am. Nat. 138, 37-50.

[25] Abrams, P. A., Matsuda, H., \& Harada, Y. (1993) Evolutionarily unstable fitness maxima and stable fitness minima of continuous traits. Evol. Ecol. 7, 465-487.

[26] Ridley, M. (1996) Evolution, 2nd Edition. Blackwell Science.

[27] Galis, F. \& Metz, J. A. J. (1998) Why are there so many cichlid species? Trends in Ecology and Evolution 13, 1-2.

[28] Dieckmann, U. \& Law, R. (1996) The dynamical theory of coevolution: A derivation from stochastic ecological processes. Journal of Mathematical Biology 34, 579-612.

[29] Geritz, S. A. H., Gyllenberg, M., Jacobs, F. J. A., \& Kalle, P. (2002) Invasion dynamics and attractor inheritance. Journal of Mathematical Biology 44, 548-560.

[30] Diekmann, O., Gyllenberg, M., Huang, H., Kirkilionis, M., Metz, J. A. J., \& Thieme, H. R. (2001) On the formulation and analysis of general deterministic structured population models: II. Nonlinear theory. J. Math. Biol. 43, 157-189.

[31] Diekmann, O., Gyllenberg, M., \& Metz, J. A. J. (2003) Steady state analysis of structured population models. Theor. Pop. Biol. 63, 309-338.

[32] MacArthur, R. \& Levins, R. (1964) Competition, habitat selection and character displacement in a patchy environment. Proc. Nat. Acad. Sci. 51, 1207-1210.

[33] Levin, S. M. (1970) Community equlibria and stability, and an extension of the competitive exclusion principle. Am. Nat. 104(939), 413-423.

[34] McGehee, R. \& Armstrong, R. A. (1997) Some mathematical problems concerning the ecological principle of competitive exclusion. J. Differ. Equations 23, 30-52. 
[35] Armstrong, R. A. \& McGehee, R. (1980) Competitive exclusion. Amer. Nat. 115(2), $151-170$.

[36] Christiansen, F. B. \& Fenchel, T. (1984) Theories of populations in biological communities. Springer.

[37] Athreya, K. B. \& Ney, P. (1972) Branching processes. Springer.

[38] Marrow, P., Dieckmann, U., \& Law, R. (1996) Evolutionary dynamics of predatorpray systems: an ecological perspectives. Journal of Mathematical Biology 34, 556578 .

[39] Maynard Smith, J. (1982) Evolution and the theory of games. Cambridge University Press, Cambridge.

[40] Meszéna, G., Kisdi, É., Dieckmann, U., Geritz, S. A. H., \& Metz, J. A. J. (2001) Evolutionary optimisation models and matrix games in the unified perspective of adaptive dynamics. Selection 2, 193-210.

[41] Bailey, N. T. J. (1964) The elements of stochastic processes. John Wiley and Sons.

[42] Goel, N. S. \& Richer-Dyn, N. (1974) Stochastic models in biology. Academic Press.

[43] Geritz, S. A. H., van der Meijden, E., \& Metz, A. J., Johan (1999) Evolutionary dynamics of seed size and seedling competitive ability. Theoretical Population Biology 55, 324-343.

[44] Meszéna, G., Czibula, I., \& Geritz, S. A. H. (1997) Adaptive dynamics in a 2-patch environment: a toy model for allopatric and parapatric speciation. J. Biol. Syst 5, $265-284$.

[45] Kisdi, É. \& Geritz, S. A. H. (1999) Adaptive dynamics in allele space: evolution of genetic polymorphism by small mutations in a heterogeneous environment. Evolution 53, 993-1008.

[46] Mathias, A., Kisdi, É., \& Olivieri, I. (2001) Divergent evolution of dispersal in a heterogeneous landscape. Evolution 55, 246-259.

[47] Kisdi, É. (2002) Dispersal: Risk spreading versus local adaptation. American Naturalist 159, 579-596.

[48] Meszéna, G. \& Szathmáry, E. (2001) Adaptive dynamics of parabolic replicators. Selection 2, 147-159.

[49] Gould, S. J. \& Eldredge, N. (1977) Punctuated equilibria: the tempo and mode of evolution reconsidered $\mathbf{3}, 115-151$.

[50] MacArthur, R. \& Levins, R. (1967) The limiting similarity, convergence, and divergence of coexisting species. Am. Nat. 101, 921, 377-385.

[51] Christiansen, F. B. (1988) Frequency dependence and competition. Phil. Trans. R. Soc. Lond. B 319, 587-600.

[52] Christiansen, F. B. \& Loeschce, V. (1980) Evolution and intraspecific exploitative competition i. one-locus theory for small additive gene effects. Theor. Pop. Biol. 18, 3, 297-313. 
[53] Loeschce, V. \& Christiansen, F. B. (1984) Evolution and intraspecific competition. ii. a two-locus model for additive gene effects. Theor. Pop. Biol. 26, 228-264.

[54] Christiansen, F. B. \& Loeschce, V. (1987) Evolution and intraspecific competiton. iii. one-locus theory for small additive gene effects and multidimensional resource qualities. Theor. Pop. Biol. 31, 33-46.

[55] Eigen, M. (1971) Molecular selforganization and the early stages of evolution. Quarterly Review of Biophysics 4, 149-212.

[56] Eigen, M., McCaskill, P., \& Schuster, P. (1989) The molecular qusispecies. Adv. Chemical Physics 75, 149-263.

[57] Eigen, M. \& Schuster, P. (1977) The hypercycle: A principle of natural selforganization: Part a: emergence of hypercycle. Naturwissenshaften 64, 541-565.

[58] Baake, E. \& Gabriel, W. (2000) Biological evolution through mutation, selection and drift: An introductory review. Ann. Rev. Comp. Phys. VII, 203-264.

[59] Drossel, B. (2001) Biological evolution and statistical physics. Advances in Physics 50, 209-295.

[60] Amitrano, C., Peliti, L., \& Saber, M. (1989) Population dynamics in a spin-glass. model of chemical evolution. Journal of Molecular Evolution 29, 513-525.

[61] Peliti, L. (2002) Quasispecies evolution in general mean-field landscapes. Europhysics Letters 57, 745-751.

[62] Mayr, E. (1947) Evolution and the Diversity of Life Selected Essays. The Belknap Press of Harvard University Press, Cambridge, Massachusetts and London, England.

[63] Gavrilets, S. (1997) Evolution and speciation on holey adaptive landscapes. Trends in Ecology and Evolution 12, 307-312.

[64] Schluter, D. (2001) Ecology and the origin of species. Trends in Ecology 85 Evolution 16, $7,372-380$.

[65] Schliewen, U. K., Tautz, D., \& Pääbo, S. (1994) Sympatric speciation suggested by monophyly of crater lake cichlids. Nature 368, 623-629.

[66] Via, S. (2001) Sympatric speciation in animals: the ugly duckling grows up. Trends in Ecology \& Evolution 16, 7, 381-390. 